\title{
Exploring the High-Pressure Materials Genome
}

\author{
Maximilian Amsler, ${ }^{1, *}$ Vinay I. Hegde, ${ }^{1, \dagger}$ Steven D. Jacobsen, ${ }^{2, \$}$ and Chris Wolverton ${ }^{1, \S}$ \\ ${ }^{1}$ Department of Materials Science and Engineering, Northwestern University, \\ Evanston, Illinois 60208, USA \\ ${ }^{2}$ Department of Earth and Planetary Sciences, Northwestern University, Evanston, Illinois 60208, USA
}

(Received 20 March 2018; revised manuscript received 23 September 2018; published 9 November 2018)

\begin{abstract}
A thorough in situ characterization of materials at extreme conditions is challenging, and computational tools such as crystal structural search methods in combination with $a b$ initio calculations are widely used to guide experiments by predicting the composition, structure, and properties of high-pressure compounds. However, such techniques are usually computationally expensive and not suitable for largescale combinatorial exploration. On the other hand, data-driven computational approaches using large materials databases are useful for the analysis of energetics and stability of hundreds of thousands of compounds, but their utility for materials discovery is largely limited to idealized conditions of zero temperature and pressure. Here, we present a novel framework combining the two computational approaches, using a simple linear approximation to the enthalpy of a compound in conjunction with ambient-conditions data currently available in high-throughput databases of calculated materials properties. We demonstrate its utility by explaining the occurrence of phases in nature that are not ground states at ambient conditions and by estimating the pressures at which such ambient-metastable phases become thermodynamically accessible, as well as guiding the exploration of ambient-immiscible binary systems via sophisticated structural search methods to discover new high-pressure phases.
\end{abstract}

DOI: 10.1103/PhysRevX.8.041021

\section{INTRODUCTION}

The laws of thermodynamics dictate that only compounds corresponding to global minima of the Gibbs free energy for a given set of external conditions are viable ground states with infinite lifetimes [1]. For such materials, there always exists a synthetic route that follows an overall exothermic chemical reaction pathway, and all systems at finite temperature will ultimately attain a Boltzmann distribution with a high occupation of the ground state in thermodynamic equilibrium. In practice, however, materials in many industrially relevant applications are metastable; i.e., they have higher energies than the equilibrium ground states. Such metastable phases, or polymorphs,

\footnotetext{
*Present address: Laboratory of Atomic and Solid State Physics, Cornell University, Ithaca, New York 14853, USA. amsler.max@gmail.com

†hegde@u.northwestern.edu

\$s-jacobsen@northwestern.edu

§c-woverton@northwestern.edu

Published by the American Physical Society under the terms of the Creative Commons Attribution 4.0 International license. Further distribution of this work must maintain attribution to the author(s) and the published article's title, journal citation, and DOI.
}

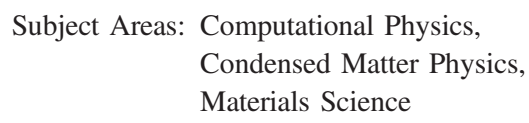

correspond to local minima on the energy landscape and are surrounded by sufficiently high barriers to render them kinetically persistent on a finite timescale [2,3].

Synthesizing metastable materials essentially requires finding, in some manner, a path in configurational space such that precursors undergo chemical reactions along a downhill trajectory with sufficiently low activation barriers, until the desired product is formed and quenched [4,5]. A plethora of thermodynamic parameters can be tuned to design such a pathway, including temperature, pressure, electromagnetic fields, compositional variations, choosing specific precursor materials, etc. A special case of this design procedure is to choose a set of thermodynamic parameters such that the desired phase becomes the thermodynamic ground state at the chosen conditions, where it forms at equilibrium and can be recovered as a metastable phase at ambient conditions if all transition barriers leading away from it are sufficiently high [6].

This problem of identifying the ground states for a given set of external conditions is commonly tackled in the computational materials-discovery community through global optimization of a target fitness function, using advanced crystal structure prediction (CSP) methods [7]. Ideally, this fitness function corresponds to the Gibbs free energy, but it is often approximated by the potential energy 
(at zero pressure and temperature) or the enthalpy (at zero temperature) or some other biased energy landscape, and it is sampled in an unconstrained manner in configurational space. Many novel materials and their structures have been resolved using CSP at high pressures [8-17], using chemical pressure and thermal degassing [18,19], as twodimensional materials [20-22], or at surfaces and interfaces [23-27]. However, CSP approaches are computationally demanding, and their applications are therefore often limited to small subsets of chemical spaces.

On the other hand, data-driven approaches using large materials databases in conjunction with high-throughput (HT) density functional theory (DFT) calculations have become increasingly popular in materials science [28-33]. Such HT databases usually contain DFT-calculated properties such as formation energy, equilibrium volume, and relaxed atomic coordinates for experimentally reported phases available in repositories such as the Inorganic Crystal Structure Database (ICSD) [34]. These data sets are sometimes augmented with hypothetical compounds constructed by decorating common structural prototypes with elements in the periodic table. Subsequent phase stability analysis is often performed to identify stable phases in every chemical space. Although approaches using such HT-DFT databases are useful for efficient large-scale analysis of energetics across a wide range of chemistries, they lack the power to predict novel materials with unknown crystal structures and phases beyond ambient conditions since all such databases currently contain only materials properties calculated at zero temperature and zero pressure.

In particular, vast unexplored pressure-composition space is becoming increasingly accessible through largevolume press and diamond anvil cell (DAC) techniques [35], and improving predictive methods for high-pressure phases is, e.g., relevant to geophysical studies of planetary interiors where there can be numerous polymorphs energetically in close proximity, even in relatively simple compositional systems [36]. Increasingly, many phases created at high pressure can be recovered to ambient conditions (e.g., diamond, silicate perovskite, etc.), where they survive as metastable materials. Furthermore, materials at high pressures show remarkable properties such as exotic stoichiometries and physics, high-temperature superconductivity, high energy density, superhardness, etc. [11]. Thus, a framework to rapidly assess phase stability and predict new materials at high pressure has the potential for a broad impact ranging from geophysical sciences to uncovering new materials physics.

In this work, we effectively combine big data in HT-DFT databases with CSP methods to unravel the "high-pressure fingerprint" of all materials and discover novel materials that are stable at nonambient pressure conditions. Using the implicitly available high-pressure information in a HT-DFT database, the Open Quantum Materials Database
(OQMD), together with a simple approximation to the formation enthalpy of a compound, we study the effect of pressure on the thermodynamic scale of stability or metastability of inorganic compounds. Our model correctly predicts most experimentally reported high-pressure elemental and binary phases to become thermodynamically stable at nonambient pressures. Our statistical analysis of the data in the OQMD shows a large fraction (up to 60\%) of ambient-metastable compounds to be thermodynamic ground states at nonzero pressures. Further, we use our model to sample all binary intermetallic chemical spaces with no experimentally reported compound in the OQMD (about 1780 chemical spaces) and predict nearly 3800 new compounds to be stable at some finite pressure. Finally, we demonstrate the power of our predictive framework in guiding sophisticated CSP methods by explicitly exploring ten binary-immiscible systems, and discover that our model correctly predicts phase spaces containing novel highpressure materials, which could be potentially recovered to ambient conditions as metastable compounds.

\section{A. Linear approximation to enthalpy}

The thermodynamic stability of solids determined by the Gibbs free energy $G=E+p V-T S$ (where $p$ and $T$ are pressure and temperature, while $E, V$, and $S$ are the internal energy, volume, and entropy of a phase) is predominantly affected by entropic effects $(T S)$ at ambient pressure since the energy scale of the $p V$ term is rather small: $p \Delta V$ at atmospheric pressure $(p=0.0001 \mathrm{GPa})$ is merely $0.006 \mathrm{meV} /$ atom even for a volume change of $\Delta V=10 \AA^{3} /$ atom. However, this behavior shifts rapidly with pressures in the GPa range, where the effect of $p V$ increases by orders of magnitude compared to entropic contributions. Hence, at zero temperature (neglecting $T S$ ), the Gibbs free energy for a given phase reduces to the enthalpy $H=E+p V$. Expanding $H$ as a function of $p$ around the equilibrium pressure $p_{0}$ yields

$$
\begin{aligned}
H(p) & =H\left(p_{0}\right)+\Delta p H^{\prime}\left(p_{0}\right)+\frac{(\Delta p)^{2}}{2} H^{\prime \prime}\left(p_{0}\right)+\cdots \\
& =H\left(p_{0}\right)+\Delta p V\left(p_{0}\right)-\frac{(\Delta p)^{2}}{2} \frac{V\left(p_{0}\right)}{B\left(p_{0}\right)}+\cdots,
\end{aligned}
$$

where $\Delta p=\left(p-p_{0}\right)$, and $B=(1 / \beta)$ is the bulk modulus of the phase, where $\beta=-(1 / V)[(\partial V) /(\partial p)]$ is its compressibility. If we neglect all terms higher than second order and consider all phases to be incompressible [i.e., $B\left(p_{0}\right) \rightarrow \infty$ ], for equilibrium pressure $p_{0}=0$, we can approximate the enthalpy of a phase simply as

$$
H(p)=E_{0}+\Delta p V\left(p_{0}\right),
$$

where $E_{0}$ is the internal energy at the equilibrium volume $V_{0}$. This approximation is used in CSP approaches to 
quickly screen large sets of generated candidate structures and was first mentioned by Pickard and Needs [8]. Conveniently, both $E_{0}$ and $V_{0}$ are quantities that are also readily available for hundreds of thousands of phases in most HT-DFT materials databases such as the OQMD [28,29], Materials Project [30], and AFLOWlib [31].

The above linear approximation to enthalpy (henceforth referred to as "LAE") is illustrated in an energy-volume diagram in Fig. 1(a), where the ground state and two metastable states are each represented by their respective equations of state (EOS) $E(V)$, i.e., their energy as a function of volume, approximated by parabolas. The negative slopes of the common tangents connecting the EOS of neighboring phases represent the pressure at which both phases are in equilibrium ("transition pressures," grey dashed lines). With our approximation of the bulk moduli $B\left(p_{0}\right) \rightarrow \infty$, the EOS curve of each phase would have infinitely large curvature, reducing the parabola to a vertical line originating at the corresponding equilibrium volumes $V_{0}$ and energies $E_{0}$. Essentially, all information of each phase is then contained in a single point at $\left(V_{0}, E_{0}\right)$, represented by filled circles.

Although the LAE is rather crude, it is reasonably accurate up to pressures in the range of tens, or even hundreds, of GPa. As we will show in the rest of this work, the LAE can be used as a powerful tool to enable quick analyses of phase stability of a large number of materials at nonambient pressures. Note that we will hereafter use the terms "zero pressure" and "ambient pressure" interchangeably since the $p V$ contribution to the free energy at atmospheric pressure is insignificant for most inorganic compounds, as discussed earlier. For example, at one atmosphere, the energy contribution of $p V$ in diamond silicon with a volume of about $20 \AA^{3}$ /atom is merely $0.012 \mathrm{meV} /$ atom, far smaller than the error bars encountered in DFT calculations.

\section{B. Thermodynamic stability: The convex hull}

The thermodynamic stability of a phase at zero temperature can be determined by the construction of the so-called convex hull of all phases in the chemical space. At zero pressure, the convex hull is constructed from the composition and formation energy (composition-energy hull, or simply " $N-E$ convex hull") of all the phases. By definition, a phase on the convex hull has a formation energy lower than that of any other phase (or linear combination of phases) at that composition and is therefore thermodynamically stable. At nonambient pressures, thermodynamic stability is determined by a convex hull which also takes into account the energy as a function of volume of all phases, given by their respective EOS, $E(V)$. The LAE introduced in Sec. I A allows us to simplify the construction of the convex hull by taking into account the ambient volume of each phase, in addition to their composition and formation energy (composition-volume-energy hull, or simply " $N-V-E$ convex hull"). A phase on the extended $N-V-E$ hull has a formation energy lower than any other phase or combination of phases at that composition and volume, and is therefore thermodynamically stable at some pressure. Furthermore, a tie-line on the convex hull represents a two-phase equilibrium, a triangular facet represents a three-phase equilibrium, and so on- $\mathrm{a}$ facet with $n$ vertices represents an $n$-phase equilibrium. (a)

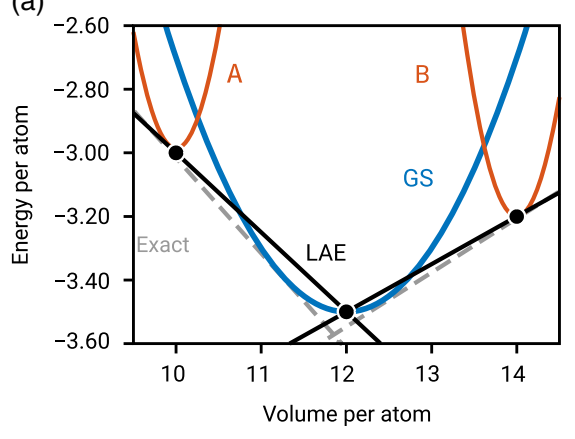

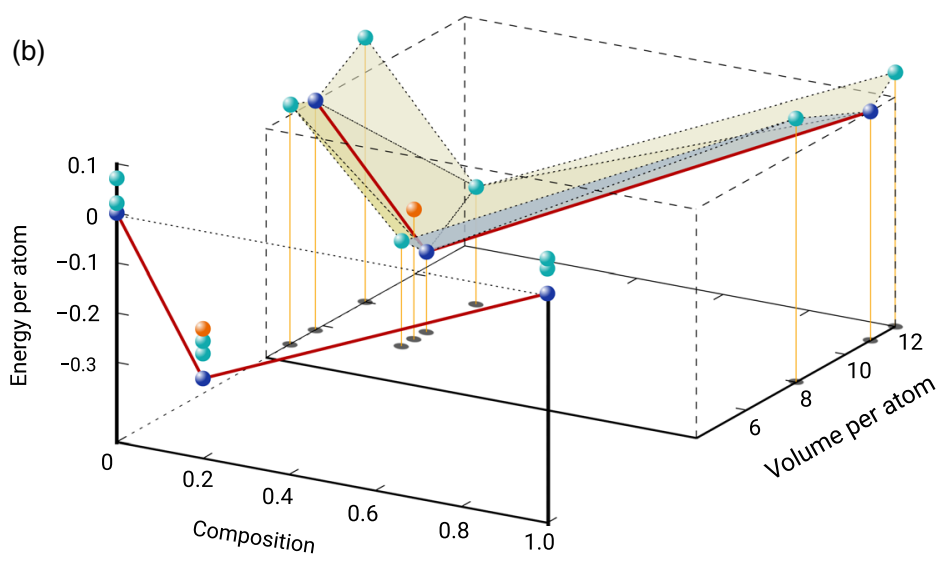

FIG. 1. (a) A schematic energy-volume ( $E-V)$ diagram with three phases [the ground state (GS), and two metastable phases A and B] and their EOS, each represented by a parabola. The negative slope of the common tangent to two adjacent EOS (dashed grey lines) represents the pressure at which the two phases are in equilibrium. The LAE approximates the common tangent with a line connecting the ambient-condition equilibrium volumes and energies of two adjacent phases (solid black line connecting filled circles). (b) A schematic $N-V-E$ convex hull for a model binary system. Individual phases are represented by spheres, and convex hull boundaries are indicated with solid red and dotted black lines. On the left is the conventional zero-pressure $N-E$ hull, a projection of the extended $N-V-E$ convex hull on the right. Phases that are thermodynamically stable at zero pressure lie on the $N-E$ convex hull (blue spheres). Metastable phases that are stable at some nonambient pressure lie above the $N-E$ hull but on the $N-V-E$ convex hull (teal spheres). A phase that is truly unstable at any pressure lies above the $N-V-E$ hull (orange sphere). 
A schematic $N-V-E$ convex hull is shown in Fig. 1(b). A projection of the extended $N-V-E$ convex hull taking into account only the compositions and energies leads to the $N-E$ hull (indicated by solid red lines). Phases that lie above the $N-E$ hull, but on the $N-V-E$ hull, are metastable at zero pressure but thermodynamically stable at some finite pressure. For example, in Fig. 1(b), only two elemental phases and one binary compound (blue spheres) lie on the $N-E$ hull (solid red lines), i.e., are thermodynamically stable at zero pressure, and all other phases are metastable. However, all elemental phases and all binary compounds except one lie on the extended $N-V-E$ hull (teal spheres connected by dotted black lines), i.e., are thermodynamically stable at some nonambient pressure. Only one phase shown (orange sphere at composition 0.2) is truly unstable at all pressures.

\section{Pressure range of stability}

For a system in thermodynamic equilibrium at zero temperature, $d E=-p d V+\sum_{i} \mu_{i} d N_{i}$, where $d E$ and $d V$ are infinitesimal changes in internal energy $E$ and volume $V$ of the system, respectively, and $d N_{i}$ is the infinitesimal change in the composition $N_{i}$ of species $i$. The equilibrium pressure is thus given by $p=-[(\partial E) /(\partial V)]_{N_{i}}$, i.e., the derivative of energy with respect to volume at constant composition. Hence, the pressure range of stability of a phase $\mathcal{P}$ with ambient equilibrium volume and energy of $V_{0}$ and $E_{0}$, respectively, is governed by the phase equilibria at volumes $\left(V_{0}+d V\right)$ and $\left(V_{0}-d V\right)$ [37]. In other words, the window of pressures $\left[p_{-}, p_{+}\right]$, where $\mathcal{P}$ is stable, is given by

$$
p_{ \pm}=-\frac{E_{0}-E\left(V_{0} \mp d V\right)}{d V} .
$$

Note that $E\left(V_{0} \pm d V\right)$ can be calculated by minimizing the free energy of the system at the target composition and volume. Grand canonical linear programming (GCLP) [38] techniques using efficient linear solvers are routinely employed to calculate phase stabilities and equilibrium reaction pathways at $0 \mathrm{~K}$ and $0 \mathrm{GPa}$ [39-43]. In this work, in addition to the average composition of the system being constrained to that of $\mathcal{P}$, the volume is constrained to $V_{0} \pm d V$ during energy minimization. Thus, a pressure range of stability can be calculated for every phase that lies on the extended $N-V-E$ convex hull.

As discussed in Sec. IA, the negative slope of the common tangent to the EOS of two phases is the pressure at which the respective phases coexist; in other words, one phase transforms into the other under the effect of pressure. In the LAE, the common tangent is reduced to a line connecting the local minima of the two phases [solid black line connecting filled circles in Fig. 1(a)]. The LAE introduces errors compared to the real transition pressure, which depend on the overall features of the energy landscape. If we assume that all phases are compressible with identical, finite bulk moduli, the LAE will consistently lead to an underestimation of the magnitude of the transition pressures. In practice, however, high-pressure phases often exhibit shorter, stronger bonds that lead to higher bulk moduli. Hence, the LAE would lead to a better agreement with the real transition pressures for phases that are stable at very high pressures. On the other hand, if the bulk moduli significantly decrease with pressure, the LAE would lead to an overestimation of the magnitude of the transition pressures. We also note that transition pressures, based on the above definition, can be positive or negative [e.g., the common tangents connecting the GS with metastable phases A and B, respectively, in Fig. 1(a)]. A negative pressure can be physically interpreted as a tensile stress, leading to the expansion of a phase toward volumes exceeding its ambient ground-state equilibrium volume.

\section{RESULTS AND DISCUSSION}

\section{A. Model assessment}

We first evaluate the accuracy of the linear approximation to enthalpy by investigating two elements and five binary systems in detail.

\section{Elemental solids}

We choose two elements whose high-pressure phase diagrams are among the most complex as well as the most well studied: silicon and bismuth. Both elements have intricate energy landscapes with several high-pressure allotropes.

Silicon.-The phase diagram of silicon has been well explored experimentally, partially due to its importance in the semiconductor industry. The ambient ground state is Si-I, which crystallizes in a cubic diamond structure [44]. It transforms around $11 \mathrm{GPa}$ to the $\mathrm{Si}$-II phase, which has a $\beta$-Sn structure type [45]. This is followed by a transformation to Si-XI with Imma symmetry [46] at $13 \mathrm{GPa}$. Above $16 \mathrm{GPa}, \mathrm{Si}-\mathrm{V}$ forms in the simple hexagonal structure [47], and at $38 \mathrm{GPa}$, Si-VI forms in an orthorhombic $\mathrm{Cmcm}$ structure [48]. The hexagonal close-packed Si-VII forms above $42 \mathrm{GPa}$ [49], and finally, cubic closepacked $\mathrm{Si}-\mathrm{X}$ forms at pressures above $78 \mathrm{GPa}$ [50].

We first compute the pressure range of stability of the various silicon allotropes using DFT calculations [see the top bar labeled "DFT" in Fig. 2(a)]. For each phase, we calculate the enthalpy explicitly by relaxing the atomic coordinates and cell parameters at various pressures in intervals of $2 \mathrm{GPa}$ and $10 \mathrm{GPa}$ in the ranges of $0-20 \mathrm{GPa}$ and 20-100 GPa, respectively. The transition pressures are then computed by minimizing the interpolated formation enthalpies as a function of pressure. This approach is more accurate than fitting an EOS using energies computed at scaled volumes since (a) the EOS fit depends on the functional form (e.g., Vinet, Murnaghan, 
Birch-Murnaghan, etc.) and (b) the simple scaling of volume assumes isotropic compressibility. We employ the above approach of explicit enthalpy calculation for all phase diagrams denoted hereafter with "DFT".

The experimentally reported sequences of formation and transition pressures of high-pressure $\mathrm{Si}$ allotropes are well reproduced, with the exception of $\mathrm{Si}$-II, which is effectively degenerate in enthalpy to Si-XI. The discrepancy between experiment and theory for the transition from $\mathrm{Si}$-I to Si-II has been well studied $[60,61]$ and is attributed to the errors associated with the PBE approximation to the DFT exchange-correlation potential.

We then calculate the pressure range of stability of all the allotropes using only the respective equilibrium energies and volumes at $0 \mathrm{GPa}$, extrapolated linearly as described in Secs. I A-IC [see the second bar labeled "LAE" in Fig. 2(a)]. The agreement between the DFT and LAE phase diagrams is remarkable: (a) The sequence of the phases is correctly reproduced, with the only exception being Si-X, which the linear approximation model predicts to be unstable even at $100 \mathrm{GPa}$, and (b) the overall errors in the transition pressures predicted by the approximate model are within around $10 \%$ of those calculated explicitly.
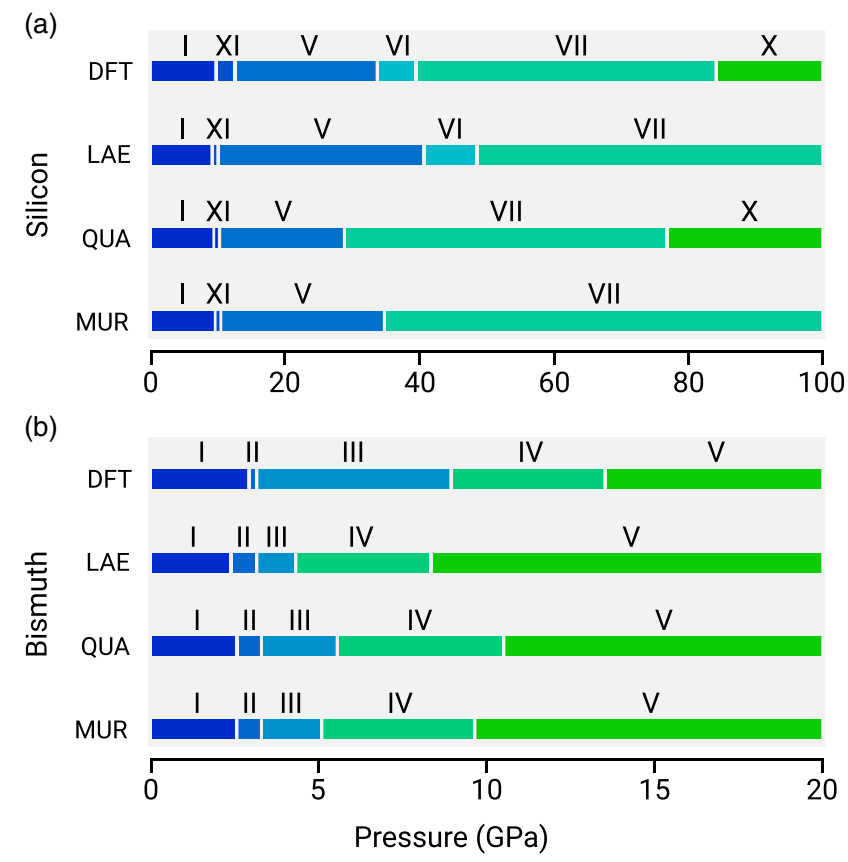

FIG. 2. The pressure range of stability of high-pressure phases of elemental (a) silicon and (b) bismuth. Explicitly computed transition pressures using DFT-calculated formation enthalpies are labeled "DFT" (top bar in each panel), and those based on the LAE are denoted with "LAE" (second bar in each panel). In addition, we show transition pressures calculated using the expansion of the enthalpy to second order ("QUA") and the Murnaghan EOS ("MUR") [51,52], for comparison. The crystal structures of the silicon and bismuth allotropes were taken from Refs. [44-50] and [53-59], respectively.
Additionally, we compare the results from LAE with those from fitted EOS. For this purpose, we scale the lattice vectors of every structure relaxed at $0 \mathrm{GPa}$ by factors of $x=\{0.95,0.97,0.98,0.99,1.00,1.01,1.02,1.03,1.05\}$ and compute the corresponding energies with single-point DFT calculations. The energy-volume data obtained in this way is used to fit two EOS: (a) a quadratic polynomial corresponding to the second-order expansion in Eq. (1) ("QUA," third bar) and (b) the well-known Murnaghan EOS [51,52] ("MUR," bottom bar). The pressure range of stability calculated using the EOS shows an overall trend and errors comparable to those calculated using the LAE. The quadratic fit fails to predict the stability of Si-VI, while it correctly assigns a pressure range below $100 \mathrm{GPa}$ for Si-X. The Murnaghan EOS performs even worse: It misses both the stability of Si-VI and the onset pressure of $\mathrm{Si}-\mathrm{X}$. In other words, using sophisticated EOS does not necessarily improve the predicted phase diagram compared to the LAE, especially given the additional computational cost associated with it [62].

Bismuth.-At ambient conditions, bismuth crystallizes in a rhombohedral $\mathrm{Bi}-\mathrm{I}$ phase with space group $R \overline{3} \mathrm{~m}$. It transforms at a pressure of around $2.55 \mathrm{GPa}$ to Bi-II with a $C 2 / \mathrm{m}$ structure $[53,54]$ and a very narrow range of stability at low temperatures. Upon increasing the pressure, Bi-III forms in a complicated, incommensurate host-guest structure with $P 4 / n c c$ symmetry [55-57]. A Bi-IV phase with space group $P 21 / n$ has been reported between 2.4 and $5.3 \mathrm{GPa}$ at temperatures above around $450 \mathrm{~K}$ [58]. Finally, the Bi-V bcc phase is observed at pressures above $7.7 \mathrm{GPa}$ [59].

Similar to the case of silicon, we first compute the pressure range of stability of the various bismuth allotropes using enthalpies calculated explicitly at various pressures at intervals of $1 \mathrm{GPa}$ in the range of $0-20 \mathrm{GPa}$ [see the top bar labeled "DFT" in Fig. 2(b)]. Although the experimentally reported sequence of allotropes formed is well reproduced, the transition pressures between $\mathrm{Bi}-\mathrm{III} / \mathrm{Bi}-\mathrm{IV}$ and $\mathrm{Bi}-\mathrm{IV} / \mathrm{Bi}-\mathrm{V}$ are severely overestimated. This behavior has been reported previously by Häussermann et al. [57], and corroborated in our recent work on $\mathrm{Cu}-\mathrm{Bi}$ intermetallics $[63,64]$.

The pressure range of stability of all allotropes calculated using the LAE reproduces the correct sequence of formation [see the second bar labeled "LAE" in Fig. 2(b)]. However, the agreement between the transition pressures predicted by the approximate model and those calculated explicitly are worse than that for silicon allotropes. We attribute these larger errors to the strong changes in the chemical bonds between the different bismuth phases, especially since ambient Bi-I has a layered structure, in contrast to the high-pressure phases. Hence, our approximation of equal, infinitely large bulk moduli for every phase is perhaps less reasonable for elemental phases of bismuth.

Similar to silicon, more advanced EOS fitted to energyvolume data do not significantly improve the description of 
the phase diagram. Neither the fit to a quadratic polynomial ("QUA") nor the Murnaghan EOS ("MUR") provides a much better agreement with the exact DFT transition pressures: The transition pressures between $\mathrm{Bi}$-III/Bi-IV and $\mathrm{Bi}-\mathrm{IV} / \mathrm{Bi}-\mathrm{V}$ are in a similar range as the ones from LAE. Overall, these results clearly demonstrate that the LAE can capture the general features that determine highpressure phase stability at a comparable accuracy to fits of EOS, at a significantly lower computational cost.

\section{Binary intermetallics}

When compared to pure elements, the high-pressure phase space of binary or higher-order chemical systems has been relatively unexplored experimentally. Including composition and pressure as additional degrees of freedom (d.o.f.) significantly increases the complexity of the phase space. In this section, we focus on a unique subset of chemistries: intermetallic systems of elements that are not miscible at ambient conditions but form compounds under pressure. Many of these so-called ambient-immiscible systems involve bismuth in combination with other elements. Recently, we investigated three such systems in detail, namely, $\mathrm{Fe}-\mathrm{Bi}$ [65], $\mathrm{Cu}-\mathrm{Bi}[63,64]$, and $\mathrm{Ni}-\mathrm{Bi}$ [66], by performing extensive global structure searches. Here, we use these three systems to further evaluate the performance of the linear approximation to enthalpy.
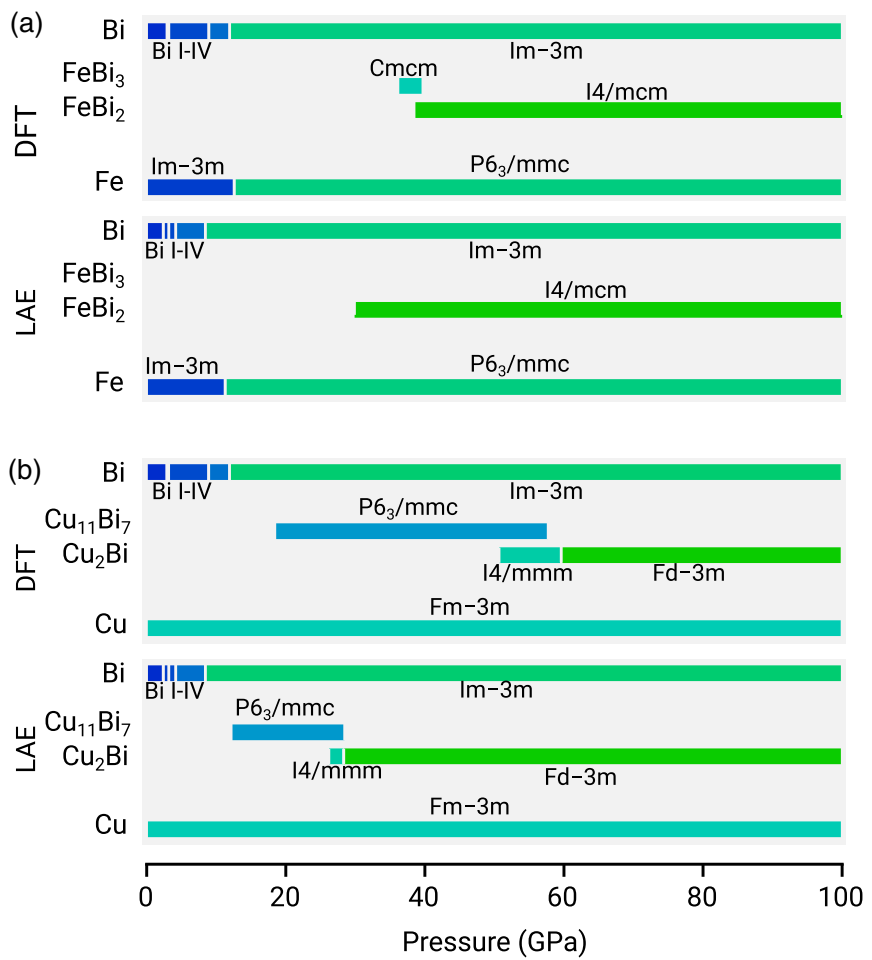

$\mathrm{Fe}$-Bi.-Using the MHM, we recently predicted a highpressure $\mathrm{FeBi}_{2}$ phase with $I 4 / \mathrm{mcm}$ symmetry at pressures above $36 \mathrm{GPa}$ [65], which was subsequently experimentally confirmed through evidence found in the in situ x-ray diffraction pattern at above $30 \mathrm{GPa}$ [10]. We note that the discovery of $\mathrm{FeBi}_{2}$ resulted from extensive MHM structural searches performed at pressures of 0,50 , and $100 \mathrm{GPa}$. The most promising candidate structures were then relaxed at pressure intervals of $10 \mathrm{GPa}$ to compute enthalpies, which were in turn used to calculate the pressure range of stability of various phases [see top panel in Fig. 3(a)]. Besides the $\mathrm{FeBi}_{2} \mathrm{I} 4 / \mathrm{mcm}$ phase, we find a $\mathrm{FeBi}_{3}$ phase with $\mathrm{Cmcm}$ symmetry to be stable in a very small pressure window, slightly below $40 \mathrm{GPa}$. This phase has not been observed in experiment so far.

We now compare the pressure range of stability calculated explicitly above with that calculated using the linear approximation to enthalpy, using only the ambient equilibrium energies and volumes of the phases. The phase diagram predicted by the approximate model [bottom panel in Fig. 3(a)] is qualitatively similar to the exact one: The $\mathrm{FeBi}_{2} \mathrm{I} 4 / \mathrm{mcm}$ phase becomes stable at comparable pressures. This finding can be conveniently exploited in structural searches: Since the MHM samples many lowlying metastable structures at a fixed pressure $p_{0}$, one could use the energies and volumes at $p_{0}$ of such phases within the LAE to quickly predict if any of the metastable phases

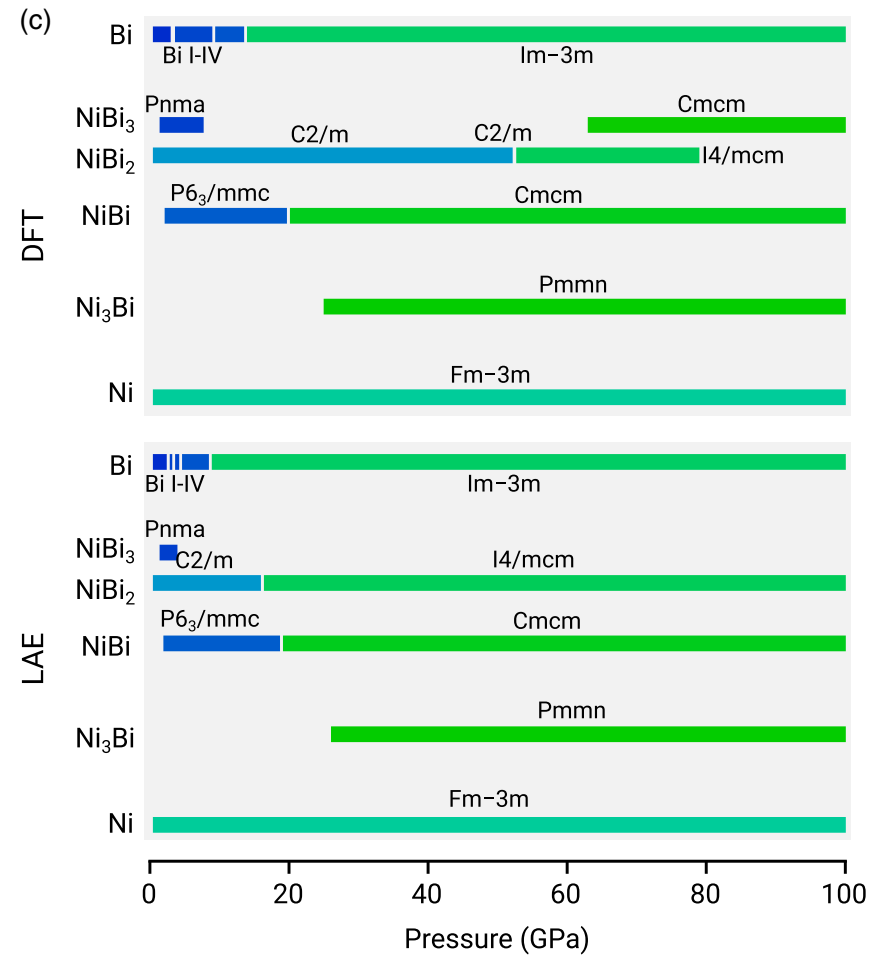

FIG. 3. Comparison between the explicitly computed phase diagrams with the ones derived from the LAE model for binary systems. Panels (a)-(c) correspond to the $\mathrm{Fe}-\mathrm{Bi}, \mathrm{Cu}-\mathrm{Bi}$, and $\mathrm{Ni}-\mathrm{Bi}$ systems, respectively. Explicitly calculated transition pressures using DFT are denoted with "DFT" (top bar), and results based on the LAE are denoted with "LAE" (bottom bar). 
become stable at a different pressure $p \neq p_{0}$. Even for immiscible systems at $p_{0}$, potential candidate structures are found if the simulation cells are sufficiently small to prevent phase segregation. This means that a structural search conducted solely at $0 \mathrm{GPa}$ might have been sufficient to uncover the $I 4 / \mathrm{mcm}$ structure and correctly predict the experimentally observed $\mathrm{FeBi}_{2}$ phase. The $\mathrm{FeBi}_{3}$ phase, on the other hand, with the narrow pressure window of stability, is predicted to be unstable at all pressures by the approximate model; this behavior can be attributed to the approximations inherent to the LAE. The overall good agreement between the exact and approximate phase diagrams is, however, rather surprising: $\mathrm{FeBi}_{2}$ undergoes a series of magnetic transitions between 0 and $40 \mathrm{GPa}$, accompanied by abrupt changes in the unit cell volume [65], all of which are neglected in the linear approximation to enthalpy.

$\mathrm{Cu}-\mathrm{Bi}$.- In the ambient-immiscible $\mathrm{Cu}-\mathrm{Bi}$ system, at least two compounds, with compositions $\mathrm{Cu}_{11} \mathrm{Bi}_{7}$ and $\mathrm{CuBi}$, have been recently discovered in DAC experiments between 3 and $6 \mathrm{GPa}[63,64]$. Both phases can be recovered to ambient conditions and exhibit exciting superconducting and structural properties. For example, $\mathrm{CuBi}$ has a layered structure, rather uncommon for high-pressure phases, and is calculated to have an extremely low energy-cost associated with exfoliation from bulk into single sheets [67]. Further, more recent structural searches predict additional, dense $\mathrm{Cu}_{2} \mathrm{Bi}$ phases to become thermodynamically accessible at pressures above $50 \mathrm{GPa}$ [68] (recent, unpublished results show that a $\mathrm{Cu}_{7} \mathrm{Bi}_{2}$ phase with $C 2 / m$ symmetry might be even lower in enthalpy. However, this structure has not been included in the current phase analysis and will be published separately).

The top panel in Fig. 3(b) shows the pressure range of stability of the various high-pressure $\mathrm{Cu}-\mathrm{Bi}$ phases computed using explicitly calculated enthalpies for each phase. The CuBi phase is not thermodynamically stable at any pressure at zero temperature, consistent with recent reports of vibrational entropy playing a crucial role in rendering this phase synthesizable [64]. The $\mathrm{Cu}_{11} \mathrm{Bi}_{7}$ phase is thermodynamically accessible at high pressures up to around $60 \mathrm{GPa}$, when it starts to compete with two dense $\mathrm{Cu}_{2} \mathrm{Bi}$ phases [68].

The bottom panel in Fig. 3(b) shows the $\mathrm{Cu}-\mathrm{Bi}$ phase diagram computed from the LAE, using only the respective equilibrium energy and volume of each phase at $0 \mathrm{GPa}$. All phases are correctly predicted to be stable by the approximate model, consistent with the exact phase diagram. As expected, the transition pressures predicted by the approximate model are underestimated overall when compared to those calculated explicitly, presumably because of the significant structural changes in elemental bismuth as a function of pressure (see Sec. II A 1). Nonetheless, it is striking that, using the simple linear approximation to enthalpy, we could have correctly predicted all the high-pressure phases in the $\mathrm{Cu}-\mathrm{Bi}$ system from a structural search only at $0 \mathrm{GPa}$.

$N i-B i$. - We test, for the first time, the predictive power of our model by investigating the high-pressure phases in the $\mathrm{Ni}-\mathrm{Bi}$ binary intermetallic system. Two compounds have been experimentally reported at ambient pressures: $\mathrm{NiBi}$ in the hexagonal NiAs structure type [69] and $\mathrm{NiBi}_{3}$ in the orthogonal $\mathrm{RhBi}_{3}$ structure type [70,71]. Both compounds are superconductors with transition temperatures of 4.25 and 4.06 $\mathrm{K}$ in $\mathrm{NiBi}$ [72] and $\mathrm{NiBi}_{3}$ [73,74], respectively. To generate phase data to be used within the LAE to construct the convex hull and predict transition pressures, we use prototypes from our previous structural searches of the $\mathrm{Fe}-$ $\mathrm{Bi}$ and $\mathrm{Cu}-\mathrm{Bi}$ systems and substitute the $\mathrm{Fe} / \mathrm{Cu}$ sites with $\mathrm{Ni}$ atoms, followed by structural relaxations at ambient pressure. Using this ambient-pressure data set of energies and volumes, the LAE model predicts stable compounds at high pressure for the compositions $\mathrm{NiBi}_{3}$ and $\mathrm{NiBi}_{2}$. Based on this prediction, we perform a thorough investigation of the Ni-Bi system using MHM simulations at pressures of 10 and $50 \mathrm{GPa}$, which indeed reveals a number of highpressure phases.

In particular, our calculations predict new compounds that are stable at high pressure at compositions of the previously reported ambient-pressure phases, i.e., $\mathrm{NiBi}$ and $\mathrm{NiBi}_{3}$. The hexagonal $\alpha$-NiBi phase undergoes a structural transition to a TII-type structure with $\mathrm{Cmcm}$ symmetry at pressures above around $20 \mathrm{GPa}$. Similarly, the orthorhombic $\mathrm{NiBi}_{3}$ phase is thermodynamically unstable above $7.5 \mathrm{GPa}$, and a $\mathrm{Cmcm}$ structure is stable above $62 \mathrm{GPa}$. Furthermore, we discover additional stable phases at previously unexplored compositions. We find that a $\mathrm{NiBi}_{2}$ phase with $C 2 / m$ symmetry in the $\mathrm{PdBi}_{2}$ structure type is in fact thermodynamically stable at ambient pressures, a finding that was reported earlier by Bachhuber et al. [75]. At the same composition, a second $C 2 / \mathrm{m}$ phase becomes stable above $52 \mathrm{GPa}$, over a very small pressure window of less than $1 \mathrm{GPa}$, followed by a $I 4 / \mathrm{mcm}$ phase, isostructural to $\mathrm{FeBi}_{2}$. Finally, a $\mathrm{Ni}_{3} \mathrm{Bi}$ compound with Pmmn symmetry, isostructural to $\mathrm{Ni}_{3} \mathrm{Sb}$ in the $\mathrm{Cu}_{3} \mathrm{Ti}$ structure type, is predicted to be stable at pressures above $25 \mathrm{GPa}$.

One of our predictions was very recently verified by compressing $\mathrm{NiBi}$ in a DAC. Heating to temperatures above $700^{\circ} \mathrm{C}$ at pressures above approximately $28 \mathrm{GPa}$, the hexagonal $\alpha$-NiBi transforms into $\beta$-NiBi in the predicted TII structure type [66]. The experimental transition pressure is somewhat higher than the calculated value of $20 \mathrm{GPa}$. This discrepancy could be attributed to the presence of high kinetic reaction barriers in the first-order phase transition, which requires heat to induce the phase change and inevitably leads to calculated transition pressures being lower than those observed in experiment. This hypothesis is supported by detectable evidence of the $\beta$-NiBi phase in the XRD pattern upon decompression: 
The $\beta$-NiBi is kinetically persistent as low as $11.62 \mathrm{GPa}$; hence, the equilibrium pressure lies anywhere between 11.62 and $28.3 \mathrm{GPa}$. In addition, errors inherent to the approximations used in DFT calculations could also explain the difference in the observed and computed transition pressures. The approximations to the exchange-correlation potential alone can make a noticeable difference. For example, the PBE functional predicts that neither the experimentally observed $\mathrm{NiBi}$ nor the $\mathrm{NiBi}_{3}$ phase (in their reported structures) are thermodynamically stable at $0 \mathrm{GPa}$ and $0 \mathrm{~K}$. However, we find that LDA correctly places the two experimental phases on the $0 \mathrm{GPa}$ convex hull, and if we additionally take into account the vibrational entropy contributions to the free energy, $\mathrm{NiBi}_{2}$ becomes unstable at elevated temperatures. A detailed investigation of the influence of different exchange-correlation functionals and temperature effects on the calculated phase stability of $\mathrm{Ni}-\mathrm{Bi}$ compounds and their properties will be reported elsewhere.

After exploring the high-pressure $\mathrm{Ni}-\mathrm{Bi}$ system with the MHM, we a posteriori compare the phase diagram of $\mathrm{Ni}-\mathrm{Bi}$ computed using the explicitly calculated enthalpies against that predicted from our LAE model [Fig. 3(c)], and we find remarkable agreement. Most phases, and the sequence in which they form under pressure, are correctly predicted by the approximate model. The only exceptions are the $\mathrm{Cmcm}$ phase at the $\mathrm{NiBi}_{3}$ composition and the second $C 2 / m$ compound at the $\mathrm{NiBi}_{2}$ composition at around $50 \mathrm{GPa}$.

To assess the source of this discrepancy, we investigate the convex hull of the $\mathrm{Ni}-\mathrm{Bi}$ system at $70 \mathrm{GPa}$, a pressure where the $C \mathrm{mcm}$ phase of $\mathrm{NiBi}_{3}$ and the $I 4 / \mathrm{mcm}$ phase of $\mathrm{NiBi}_{2}$ overlap the most (see Fig. S4 in Ref. [76]). We see that the $\mathrm{Cmcm}$ and $I 4 / \mathrm{mcm}$ phases barely lie on the convex hull of stability, and the energy difference compared to the two-phase mixture of elemental $\mathrm{Bi}$ and the $\mathrm{Cmcm}$ phase of NiBi is extremely small. This delicate feature of the enthalpy landscape in the $\mathrm{Ni}-\mathrm{Bi}$ system is hard to capture even with higher-order approximations to the enthalpy. As an additional challenge, the $\mathrm{Cmcm} \mathrm{NiBi}_{3}$ phase has a strongly anisotropic compressibility: At $100 \mathrm{GPa}$, the lattice parameters $a, b$, and $c$ are reduced by factors of $0.813,0.830$, and 0.890 , respectively, when compared to those at $0 \mathrm{GPa}$. Therefore, neither the LAE nor any simple EOS fit can correctly reproduce the exact DFT enthalpy at high pressure - they overestimate the enthalpy, moving the phase off the convex hull. Nevertheless, we recompute the complete phase diagram of the $\mathrm{Ni}-\mathrm{Bi}$ system by fitting the Murnaghan EOS to the energies at scaled volumes of every relevant phase (see Fig. S3 in Ref. [76]). As expected, the Murnaghan EOS fails to capture the phase stability of $\mathrm{Cmcm} \mathrm{NiBi}_{3}$, similar to the LAE.

As discussed earlier, the $C 2 / m$ phase at the $\mathrm{NiBi}_{2}$ composition has a very small pressure range of stability of less than $1 \mathrm{GPa}$, so its absence in the phase diagram predicted by the LAE is not surprising. The phase diagram computed using the Murnaghan EOS misses this phase as well. In fact, similar to the $\mathrm{Cmcm} \mathrm{FeBi}_{3}$ phase that was predicted to be stable in a narrow pressure window of less than $3 \mathrm{GPa}$ but has not yet been observed experimentally, synthesis of the $\mathrm{NiBi}_{2}$ phase is likely to be challenging, if possible at all. Overall, stability predictions using the LAE and the Murnaghan EOS are very comparable, similar to our findings for the elemental phase diagrams.

\section{Binary oxides}

Next, we assess the performance of the LAE in predicting the stability and the transition pressures of phases in two ceramic oxide systems with several experimentally studied high-pressure phases: $\mathrm{Zr}-\mathrm{O}$ and $\mathrm{Ge}-\mathrm{O}$.

$\mathrm{Zr}-\mathrm{O}$.- The phase diagram of $\mathrm{Zr}-\mathrm{O}$ is complex, where the constituent elements themselves exhibit various thermodynamically stable phases as a function of pressure. Experimentally, oxygen crystallizes in the monoclinic $\alpha$ phase at ambient pressure and low temperatures [77], transforming into the orthorhombic $\delta$ phase at $3 \mathrm{GPa}$ [78], followed by the monoclinic $\epsilon$ phase at $10 \mathrm{GPa}$ $[79,80]$, and finally the $\zeta$ phase above 96 GPa through a continuous displacive and isosymmetric transition $(\epsilon-\mathrm{O}$ is essentially isostructural to $\zeta$-O) $[81,82]$. However, based on our PBE-DFT calculations, only the $\epsilon$ phase $(\zeta-\mathrm{O})$ is thermodynamically stable over the whole pressure range as we neglect magnetic ordering which persists in the condensed low-pressure regimes of $\mathrm{O}_{2}$ (see Fig. 4).

At ambient pressure, elemental $\mathrm{Zr}$ crystallizes in its low-temperature phase II, which has an hcp structure. At $2.2 \mathrm{GPa}$, a transformation to phase III with a so-called $\omega$ structure of hep type [83] is observed, and at around $30 \mathrm{GPa}, \mathrm{Zr}$ adopts a bcc structure (phase I) [84]. The top panel in Fig. 4(a) shows that our exact DFT calculations reproduce these two transitions with reasonable accuracy.

In the binary $\mathrm{Zr}-\mathrm{O}$ system, $\mathrm{ZrO}_{2}$ adopts a baddeleyitetype structure with $P 2_{1} / c$ symmetry at ambient pressure, which transforms into the orthorhombic Pbca and Pnma phases at pressures of $3 \mathrm{GPa}$ and $13-30 \mathrm{GPa}$, respectively $[85,86]$. Furthermore, the suboxide $\mathrm{Zr}_{3} \mathrm{O}$ was observed in two structure types with $R-3 c$ and $R 32$ symmetries, while the formation of $\mathrm{ZrO}$ was reported in the $\mathrm{NaCl}$ structure type with $F m-3 m$ symmetry [87]. The latter phase indeed becomes thermodynamically stable but only at pressures above approximately $45 \mathrm{GPa}$.

A comparison between the exact phase diagram of the $\mathrm{Zr}-\mathrm{O}$ system with that calculated using the LAE [Fig. 4(a)] shows good agreement. The only major discrepancy is found for $\mathrm{Zr}_{3} \mathrm{O}$ : From the explicit calculations of enthalpy, the thermodynamically stable low-pressure phase with $R-3 c$ symmetry loops back and becomes stable again, above approximately $55 \mathrm{GPa}$. By definition, such a behavior cannot be predicted within the LAE model. 

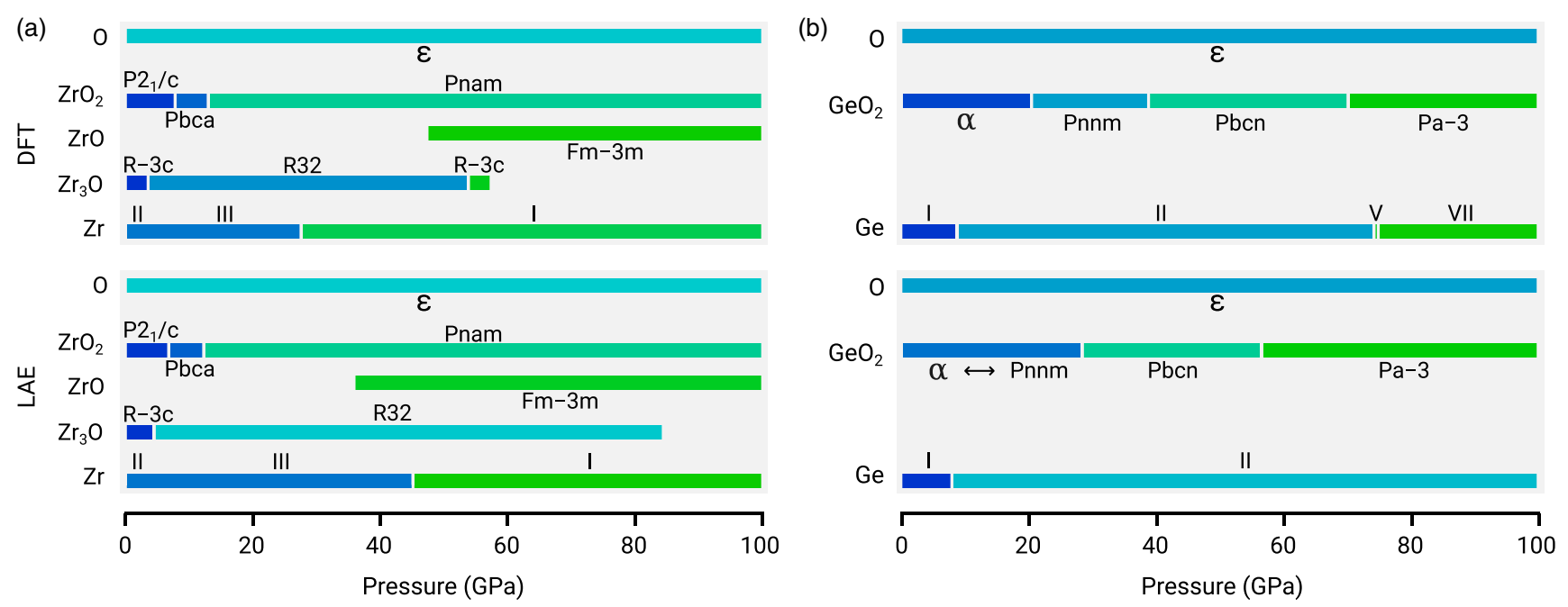

FIG. 4. Comparison between the explicitly computed phase diagrams and the ones calculated using the LAE model for two oxide systems. Panels (a) and (b) correspond to the $\mathrm{Zr}-\mathrm{O}$ and the $\mathrm{Ge}-\mathrm{O}$ systems, respectively. Explicitly calculated transition pressures using DFT are denoted with "DFT" (top bar), and results based on the LAE are denoted with "LAE" (bottom bar). The arrow denotes the displacive phase transformation.

Ge-O.-The phase diagram of elemental Ge has been extensively explored experimentally, but the exact sequence and structures of some high-pressure phases have not yet been conclusively determined. At ambient pressure, phase I adopts the diamond structure [88], which transforms to phase II with a $\beta$-Sn structure type between 6.7 and $10.5 \mathrm{GPa}$, depending on the amount of shear stress [89]. Above $74 \mathrm{GPa}$, phase $\mathrm{V}$ forms in the simple hexagonal structure [90], which transforms to an orthorhombic structure with $\mathrm{Cmcm}$ symmetry (phase VII) around $100 \mathrm{GPa}$ [91] and, subsequently, to an hep structure at 160-180 GPa (phase VIII) [92]. Additionally, a series of metastable, intermediate Ge phases have been reported [93]. The formation sequence of the high-pressure phases computed from the exact enthalpies in Fig. 4(b) agrees well with results in the literature [94]. However, the approximate LAE phase diagram incorrectly predicts phase II to be stable up to at least $100 \mathrm{GPa}$.

Similar to $\mathrm{SiO}_{2}$, there are several polymorphs of $\mathrm{GeO}_{2}$. At ambient pressure, the most stable form is the $\alpha$-quartz ground-state structure [95], which transforms to the rutiletype structure with $P 4_{2} / \mathrm{mnm}$ symmetry below $2 \mathrm{GPa}$ [96]. A displacive phase transformation to a $\mathrm{CaCl}_{2}$-type structure with Pnnm symmetry has been reported at approximately $20 \mathrm{GPa}$, followed by a transition to a $\alpha-\mathrm{PbO}_{2}$-type structure (Pbcn symmetry) above approximately $33 \mathrm{GPa}$ and a pyrite-type structure ( $\mathrm{Pa}-3$ symmetry) [97,98].

The top panel in Fig. 4(b) shows that the exact DFT results are in excellent agreement with the experimental observations. The LAE closely reproduces the exact phase diagram, with the exception of the transition from the $\mathrm{P}_{2} / \mathrm{mnm}$ phase to the Pnnm phase. This is expected as the phase transition is of second order, and a DFT relaxation of both structures at $0 \mathrm{GPa}$ results in identical equilibrium energies and volumes.

\section{B. Large-scale analysis of phase stability at high pressure}

\section{Elements and binary compounds}

The power of our linear enthalpy model lies in its capability to efficiently assess the pressure range of stability of hundreds of thousands of phases. Since the linear approximation requires only equilibrium energies and volumes of phases calculated at ambient pressure, it can be used to leverage the large materials data sets available in HT-DFT databases such as the OQMD [28,29], Materials Project [30], and AFLOWlib [31]. Here, we present large-scale analysis and statistics of thermodynamic phase stability of materials at high pressure using ambient-pressure phase data calculated in the OQMD.

First, we focus on elemental high-pressure phases and begin by compiling a "validation data set" of experimentally reported high-pressure elemental phases. The crystal structures of many high-pressure phases reported in the ICSD [34] have been calculated in the OQMD, albeit at ambient pressure. For every element, we filter all entries in the ICSD using the "External Conditions $\rightarrow$ Pressure" metadata available for each entry. Furthermore, Tonkov et al. [93] compiled a comprehensive list of phase transformations under pressure for nearly 100 elements, on which we rely heavily as a second reference to crossvalidate and augment the list of high-pressure phases calculated in the OQMD. Our final compiled data set contains 132 distinct elemental high-pressure phases and can be found in Ref. [76]. 
For each element in the periodic table, we use the ambient-pressure energy and volume data for all ICSD phases (i.e., not limited to high-pressure phases) calculated in the OQMD within the LAE model to predict (a) the number of phases from our validation data set that lie on the extended $N-V-E$ convex hull, i.e., the number of phases stable at some finite pressure, and (b) the pressure range of stability of every phase that lies on the $N-V-E$ hull. Figure 5 shows a summary of this analysis in the form of a periodic table: For every element with at least one experimentally reported high-pressure phase, we indicate the number of high-pressure phases in our compiled data set from the OQMD (bottom-left half) and the number of phases predicted by the linear enthalpy model to lie on the $N-V-E$ hull (top-right half), represented on a color scale. The number of phases reported experimentally and those predicted to be stable at some finite pressure match exactly whenever the colors in both the left and right segments are identical. This is indeed the case for most elements, with a few exceptions. Overall, $75 \%$ of all experimentally reported high-pressure phases are predicted to lie on the $N-V-E$ convex hull (see the top panel of Table I). In addition, for around 35\% of the phases, the predicted pressure range of stability overlaps with the respective transition pressures reported in experiment. The low success rate in correctly predicting the transition pressure is somewhat expected following the model validation on $\mathrm{Si}$ and $\mathrm{Bi}$ in Sec. II A. We discuss the possible sources of discrepancy between predictions and experiment toward the end of this section.

Next, we perform a similar large-scale analysis for all experimentally reported binary phases. Using calculations

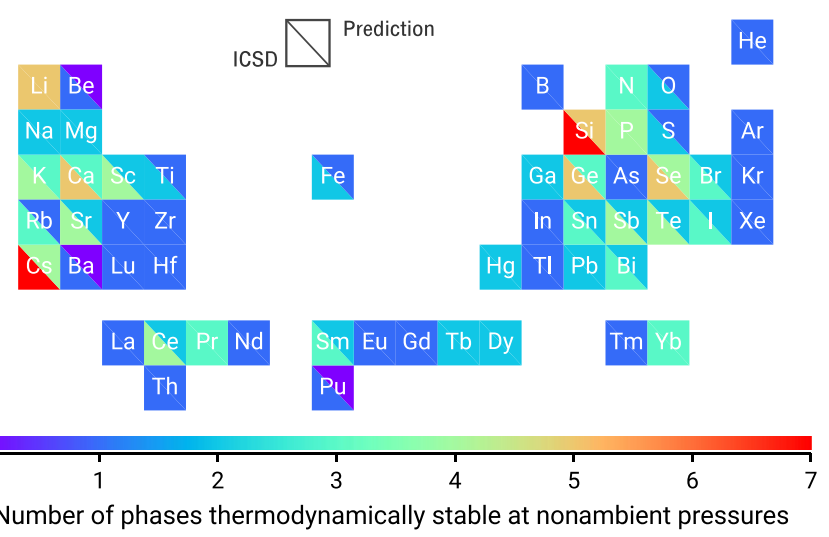

FIG. 5. Comparison of predictions of high-pressure elemental phases from the LAE model against experiment. For each element, the number of (a) unique phases reported experimentally and (b) those predicted by the linear enthalpy model to be thermodynamically stable at nonambient pressures are indicated by the color of the bottom-left and top-right halves, respectively, of the corresponding tile in the periodic table. Overall, the model correctly predicts about $75 \%$ of the high-pressure phases in the ICSD to be thermodynamically stable at nonambient pressures.
TABLE I. Accuracy of the linear enthalpy model in predicting the stability (at some finite pressure) of experimentally reported elemental and binary high-pressure phases.

\begin{tabular}{lr}
\hline \hline \multicolumn{1}{c}{ Elements } & \\
\hline $\begin{array}{lr}\text { Experimentally reported HP phases } \\
\text { Predicted to be stable at finite pressure }\end{array}$ & $97(75 \%)$ \\
$\begin{array}{l}\text { Predicted pressure range of stability } \\
\text { matches experiment }\end{array}$ & $45(35 \%)$ \\
\multicolumn{1}{c}{ Binaries } & 343 \\
\hline $\begin{array}{l}\text { Experimentally reported HP phases } \\
\text { Predicted to be stable at finite pressure } \\
\begin{array}{l}\text { Predicted pressure range of stability } \\
\text { matches experiment }\end{array}\end{array}$ \\
\hline \hline
\end{tabular}

of experimentally reported compounds in the OQMD, curated using pressure-related metadata in the ICSD (in a manner similar to that employed for elemental phases), we compile a data set of 343 unique binary compounds in total as a validation data set (the entire list is available in Ref. [76]). This number is significantly lower than that expected from a simple combinatorial estimation. For elemental solids, we find, on average, more than one high-pressure phase per element. If we extend this observation to binaries and assume that every binary system has, on average, more than one high-pressure phase, the number of potential high-pressure phases considering 90 elements is ${ }^{90} \mathrm{C}_{2}=4005$. We note that our estimation is very conservative since binary $A-B$ systems introduce an additional, compositional d.o.f., which allows multiple highpressure phases to exist at the same pressure, $A_{\mathrm{p}} B_{\mathrm{q}}$, as we have seen in Sec. II A 2. This indicates that the highpressure phase diagrams of binary systems, in general, have been relatively underexplored. The linear enthalpy model performs equally well for binary compounds-80\% of experimentally reported high-pressure binary phases are predicted to be stable at some finite pressure (see lower panel of Table I). For around 35\% of the phases, the predicted pressure range of stability overlaps with the respective transition pressures reported experimentally.

Overall, our "crude" linear enthalpy model performs surprisingly well, with a success rate of $75 \%-80 \%$, in predicting the stability of both elemental and binary highpressure phases. We identify four potential sources of error that could explain the discrepancy between the number of high-pressure phases reported experimentally and that predicted by our approximate model:

(a) The crystal structure reported experimentally for the phase is erroneous. Resolving the crystal structure, e.g., from in situ XRD measurements, under high pressure is a difficult and tedious task that can lead to incomplete or incorrect structural characterization. A prominent example is the Bi-III phase, the crystal structure of which was experimentally resolved only after several failed attempts [55]. In fact, Bi-III has an 
incommensurate host-guest structure, and the reported structure is only a representative ordered model with $P 4 / n n c$ symmetry [57]. A similar incommensurate structure has been reported for phase IV of phosphorus in the pressure range of 107-137 GPa [99].

(b) The high-pressure phase emerges via a phase transition of second order. In this case, the structural relaxations performed using DFT will inevitably transform the high-pressure phase to a lower-pressure structure. Therefore, our linear enthalpy model, which relies on the equilibrium energy $E_{0}$ and volume $V_{0}$ at ambient pressure of a high-pressure phase, will expectedly not capture its stability.

(c) There are errors inherent to DFT calculations and numerical noise, e.g., due to the approximation to the exchange-correlation potential, pseudization of core electrons (which might be important, especially at high pressures), unconverged basis sets and sampling meshes, insufficient tolerances during structural relaxations, etc.

(d) Finally, there is the inherent error due to applying a linear approximation to the enthalpy of each phase (i.e., assuming all phases to be perfectly incompressible), which might be unreasonable for some materials at large values of pressure. In particular, the LAE, like all commonly used empirical EOS, assumes isotropic compressibility, which leads to issues with strongly anisotropic phases, as we discuss in detail for the Cmcm phase of $\mathrm{NiBi}_{3}$.

One way to address some of the above limitations in the LAE for practical materials-discovery efforts is to take into account phases within a small distance $\Delta H$ off the $N-V-E$ convex hull, in addition to the ones that lie on it. For example, an enthalpic tolerance of $\Delta H=25 \mathrm{meV} /$ atom can be used to account for the influence of entropic effects at room temperature on thermodynamic phase stability [6]. Phases that are not on the hull, but sufficiently close to it, can then be included in explicit enthalpy calculations to obtain reliable predictions of accurate high-pressure phase diagrams to guide experimental synthesis.

\section{All experimentally reported compounds}

We now use our linear enthalpy model to analyze the phase stability of all experimentally reported compounds calculated in the OQMD (not limited to high-pressure phases), a total of around 33000 unique ordered compounds. As we did earlier, using the equilibrium energy and volume at ambient pressure of each phase in our data set, we predict the number, and the pressure range of stability, of all phases that lie on the extended $N-V-E$ convex hull (i.e., presumably thermodynamically stable at some finite pressure).

First, we find that only around 55\% of the 33000 compounds in our data set lie on the $N-E$ convex hull, i.e., are thermodynamically stable at ambient pressure conditions, consistent with a previous report on a similar data set from the OQMD [29]. A recent study by Sun et al. [6] on a data set of 29900 experimentally reported compounds calculated in the Materials Project also finds around 50\% \pm $4 \%$ of the phases to be metastable at ambient conditions. In the latter study, it is proposed that the observed metastable compounds are generally remnants of thermodynamic conditions where they were once the stable ground states.

We next test this hypothesis of "remnant metastability" by using pressure as a thermodynamic handle and tracking the number of metastable phases that become stable with an incremental increase or decrease in pressure, with respect to ambient conditions. Figure 6(a) shows the fraction of metastable phases as a function of positive (compressive) or negative (tensile) pressure, separated into binary, ternary, quaternary, and higher-component systems. We observe a range of trends based on our statistical analysis.

First, the number of metastable phases decreases with incremental application of both positive and negative pressures, relative to $0 \mathrm{GPa}$. In other words, a significant fraction of the ambient-metastable phases are, in fact, thermodynamically stable ground states at nonambient pressure conditions. For example, in the case of binary compounds [top left in Fig. 6(a)], the fraction of phases that are metastable at ambient pressure decreases from around 0.45 at $0 \mathrm{GPa}$ to around 0.30 at $100 \mathrm{GPa}-33 \%$ of the ambient-metastable phases are rendered thermodynamically stable at some pressure $p \in(0,100] \mathrm{GPa}$. However, in each case, a sizable fraction of metastable phases remain metastable at all pressures; i.e., they are not equilibrium ground states at any pressure [represented by horizontal dashed lines in Fig. 6(a)]. For example, around $21 \%$ of all binary phases remain metastable and cannot be accessed thermodynamically via pressure alone.

Second, the rate of decrease in the number of metastable phases (or increase in the number of metastable phases made stable) with pressure is maximum near zero and decays rapidly toward higher positive or negative pressures. This is most likely due to a bias toward small values of pressure in our compiled set of phases-after all, most compounds reported experimentally are likely observed in near-ambient conditions-but could also be due to a fundamental property of materials, namely, the density of stable ground states as a function of volume or pressure is maximum near zero pressure.

Third, we find considerable differences concerning the "character" of metastability in binary, ternary, and higher order compositional systems. We distinguish two subsets for each $n$-component data set $(n=2,3,4, \geq 5)$ polymorphs and "phase separation"-depending on whether a given phase is metastable with respect to another phase at the same composition or a combination of phases, respectively, at ambient conditions. We note that the higher the number of components present in a metastable compound, the more likely it is to phase-separate rather than 

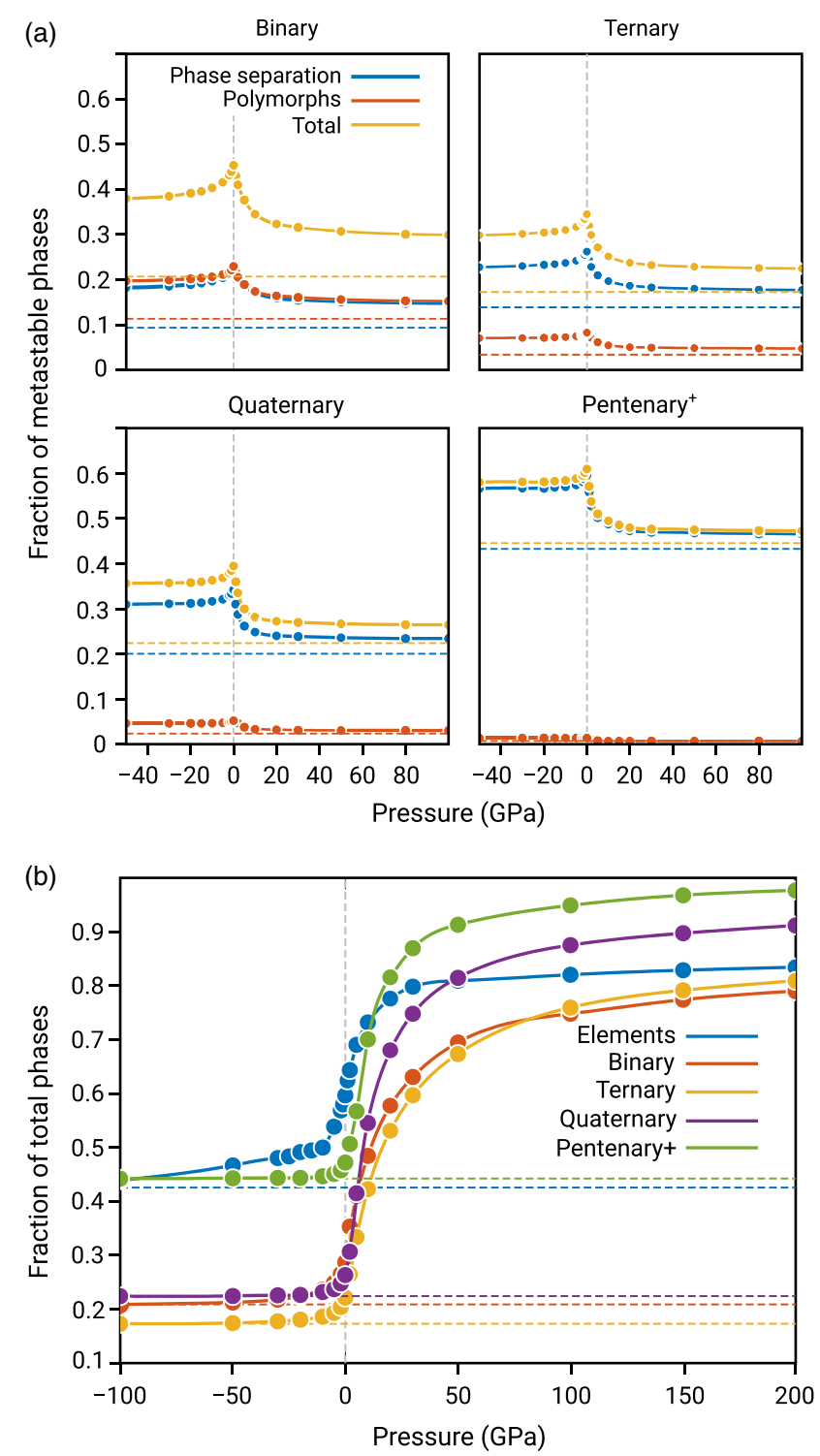

FIG. 6. (a) Fraction of metastable phases that become thermodynamically stable with incremental increase/decrease in pressure, with respect to $0 \mathrm{GPa}$. The horizontal dashed lines indicate the fraction of metastable phases that do not lie on the $N-V-E$ convex hull at any pressure. (b) Fraction of ambientmetastable phases that cannot be accessed thermodynamically at any pressure larger than pressure $p$, equivalent to 1- (fraction of phases that can be accessed at some pressure larger than pressure $p$ ).

transform into a polymorph, in agreement with previous observations [6]. Furthermore, the lower the number of components in a metastable compound, the more likely it is to be stabilized with pressure. Considering the subset of all metastable phases that phase-separate at ambient pressure, $58 \%, 47 \%, 42 \%$, and $27 \%$ become thermodynamically stable at some finite positive or negative pressure in the case of binary, ternary, quaternary, and higher-component systems, respectively.
Additionally, we observe that the effects of positive and negative pressures on the metastability of phases are not symmetric about zero pressure: A much larger portion of ambient-metastable phases become thermodynamically stable under positive (compressive) pressure when compared to negative (tensile) pressure. A difference is perhaps expected considering that the limiting behaviors are very different: Large positive pressures favor the formation of close-packed phases before eventual overlap of atomic cores, while the limit of large negative pressures is simply the individual noninteracting atoms of each species in the phase.

Finally, we probe a complementary question: If one were to incrementally tune external conditions from large positive to large negative pressures, how many observed metastable phases $\mathcal{N}$ can be accessed thermodynamically below any given pressure $p$ ? We calculate at pressure $p$ the number of experimentally observed phases from our data set that cannot be thermodynamically accessed at any pressure $>p$. We present this data as a cumulative histogram of the fraction of phases, integrated from pressures $p$ to $+\infty$, separated into elements, binaries, ternaries, etc., in Fig. 6(b). Hypothetically, if all experimentally reported compounds were thermodynamically stable ground states at some finite pressure, one would expect this cumulative fraction of unstable phases to be 1 and 0 for $p \rightarrow \infty$ and $p \rightarrow-\infty$, respectively. Consistent with our previous observations, we find that (a) a sizable fraction of the phases do not lie on the extended $N-V-E$ convex hull at all-i.e., they are not ground states under any pressure [represented by horizontal dashed lines in Fig. 6(b)] — and (b) the rate at which additional metastable phases can be thermodynamically accessed is maximum near zero pressure (given by the slopes of the curves). In other words, the pressure density of thermodynamic ground states, $(d \mathcal{N}) /(d p)$, is maximum near $p=0$. Whether this is an artifact of using a data set of experimentally observed phases or it is a fundamental property of matter needs further analysis, and this will be the subject of future work.

\section{Discovery of new high-pressure compounds}

So far, we have used the LAE to analyze the phase stability of experimentally reported high-pressure elemental and binary phases and to probe the accessibility of ambient-metastable phases using pressure as a thermodynamic handle. Now, we go a step further by using the LAE to predict new intermetallic compounds by combining it with CSP methods. For this purpose, we focus on a unique subset of binary systems, namely, the combination of elements that are immiscible at ambient pressures. According to the data we compiled from the OQMD, there currently exist about 1780 binary systems that do not contain any experimentally observed compounds. Any high-pressure phases that we identify in these systems are therefore true predictions of new materials. 
For the data set to be used for construction of the convex hull and calculation of transition pressures within the LAE, we use ambient-pressure formation energies and volumes of phases calculated in the OQMD. As mentioned in Sec. IV A, the OQMD contains calculations of more than 450000 compounds including experimentally reported compounds from the ICSD, and hypothetical compounds generated by decoration of common structural prototypes with all the elements in the periodic table. The Strukturbericht symbols of the prototype structures considered in this section are listed below $[29,100]$ :

(a) Elemental prototypes: A1 (fcc), A2 (bcc), A3 (hcp), A3' $(\alpha-\mathrm{La}), \mathrm{A} 4$ (diamond), A5 $(\beta-\mathrm{Sn}), \mathrm{A} 7(\alpha-\mathrm{As})$, A9 (graphite), A10 $(\alpha-\mathrm{Hg}), \mathrm{A} 11(\alpha-\mathrm{Ga}), \mathrm{A} 12(\alpha-\mathrm{Mn})$, $\mathrm{A} 13(\beta-\mathrm{Mn}), \mathrm{A}_{\mathrm{b}}(\beta-U), \mathrm{A}_{\mathrm{h}}(\alpha-\mathrm{Po}), C 19(\alpha-\mathrm{Sm})$.

(b) Binary $A B: \mathrm{B} 1(\mathrm{NaCl}), \mathrm{B} 2(\mathrm{CsCl}), \mathrm{B} 3$ (zincblende $\mathrm{ZnS}$ ), B4 (wurtzite $\mathrm{ZnS}$ ), B19 (AuCd), $\mathrm{B}_{h}(\mathrm{WC}), \mathrm{L1}_{0}$ $(\mathrm{AuCu}), \mathrm{L1}_{1}(\mathrm{CuPt})$.

(c) Binary $A_{3} B: \mathrm{L}_{2} \quad\left(\mathrm{Cu}_{3} \mathrm{Au}\right), \quad \mathrm{D}_{19} \quad\left(\mathrm{Ni}_{3} \mathrm{Sn}\right), \quad \mathrm{D} 0_{22}$ $\left(\mathrm{Al}_{3} \mathrm{Ti}\right), \mathrm{DO}_{3}\left(\mathrm{AlFe}_{3}\right)$.

We screen for promising chemical systems that contain high-pressure phases in the following manner: For every ambient-immiscible binary system, we use the LAE to predict the thermodynamic phase stability and pressure range of stability of each hypothetical compound in that chemical space. We select systems that contain at least one hypothetical compound predicted to become stable below an arbitrary pressure threshold of $50 \mathrm{GPa}$. We then rank these systems according to the predicted transition pressures, from lowest to highest, and select 10 of the most promising systems for further investigation. For each system, we further verify that no compound in that chemical space is reported in the ICSD or in phase diagrams available in the ASM Alloy Phase Diagram Database [101]. At each composition where our model predicts a stable high-pressure phase, we perform structural searches using the MHM, starting from the respective prototype structure from the OQMD, using simulation (super) cells with up to 10 atoms/cell. Because of the set of binary prototypes currently calculated in the OQMD (see list above), the stoichiometries we sample are limited to $A_{3} B, A B$, and $A B_{3}$. Note that both the system size and the number of sampled compositions are far too low to give accurate predictions of the true high-pressure ground states. The structural searches are merely intended as proof-ofconcept, i.e., to provide a sampling of configurations beyond the limited number of prototype structures.

Figure 7 shows the convex hulls of stability at $50 \mathrm{GPa}$ for the ten selected ambient-immiscible binary systems, namely, As-Pb, Al-Si, Sn-Bi, Fe-In, Hg-In, Hg-Sn, Re-Sn, $\mathrm{Re}-\mathrm{Br}$, Re-Ga, and $\mathrm{Zn}-\mathrm{Ga}$, after performing structural searches using the MHM. We also provide the approximate LAE convex hulls at the same pressure (extrapolated from the equilibrium volumes and energies of the prototype structures in the OQMD) in Figs. S3-S12 in Ref. [76]. For

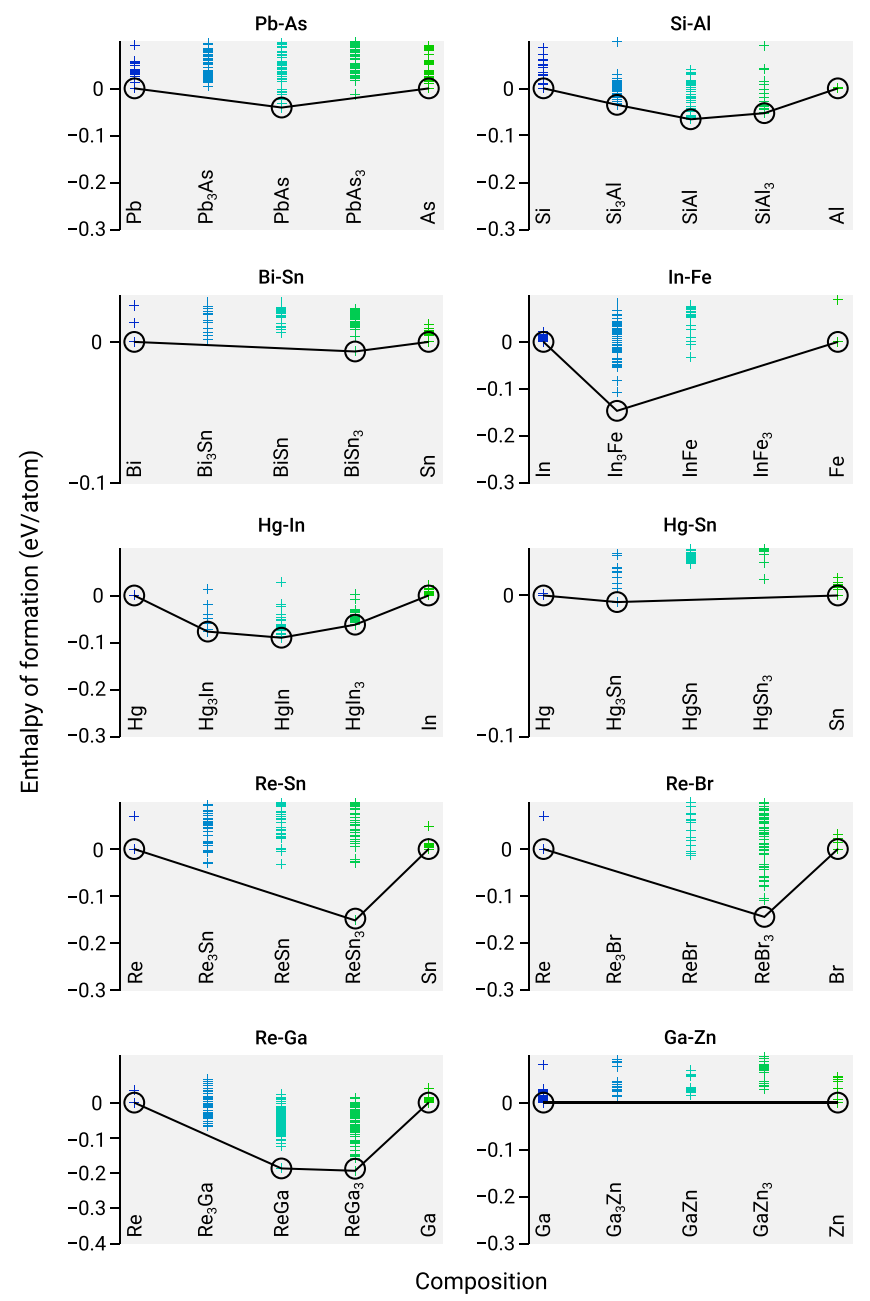

FIG. 7. The convex hulls of formation enthalpy of ten ambientimmiscible binary systems calculated using structural search at $50 \mathrm{GPa}$ via the MHM. Each cross denotes a phase sampled with the MHM. In all but the $\mathrm{Zn}-\mathrm{Ga}$ system, we find at least one thermodynamically stable high-pressure phase.

each binary system, a comparison of the MHM and LAE convex hulls shows a good agreement of the overall features. Note, however, that the ground-state structures found by the MHM calculations are different from the binary prototype structures in the OQMD, often significantly lower in enthalpy.

For all but the Zn-Ga system, the MHM searches confirm the existence of at least one new stable high-pressure phase. In fact, in nine out of the ten ambient-immiscible binary systems, the LAE correctly predicts that new compounds can be formed at sufficiently high pressures. All thermodynamically stable structures at $50 \mathrm{GPa}$ are provided in Ref. [76], together with the OQMD ID of the seed structures. Our results show that the LAE is an effective tool to identify "seed" compositions and structures for sophisticated CSP methods. The high-pressure phases predicted here present a number of avenues for experimental synthesis and verification. Overall, the success of 
the linear enthalpy model in guiding unbiased search techniques based on crystal structure prediction in discovering novel high-pressure phases is remarkable.

\section{CONCLUSIONS}

In summary, we present a method that allows an efficient screening for materials that are thermodynamically stable at nonambient pressures using a simple linear approximation to the formation enthalpy of a phase. Using a generalized convex hull construction, the stability of thousands of compounds can be evaluated at a low computational cost based on ambient-pressure data that are currently available in many materials databases without performing any additional DFT calculations. A comparison with higher-order EOS fitted to energy-volume data shows that the LAE performs similarly well at significantly lower computational cost.

Through a large-scale analysis of experimentally reported compounds, we show that a large fraction of the observed ambient-metastable phases are, in fact, thermodynamic ground states at some finite pressure. This result strongly suggests that many phases are likely remnants of highpressure conditions where they were stable ground states, even if the synthesis occurred at atmospheric pressurepotentially through local pressure fluctuations, chemical pressure, etc. Our method can be readily extended by further generalizing the convex hull construction and taking into account additional thermodynamic d.o.f., including temperature or surface areas of finite particles.

Finally, we demonstrate the predictive power of this model when combined with a crystal structure prediction technique by discovering novel high-pressure phases in a set of ambient-immiscible binary intermetallic systems.

\section{METHODS}

\section{A. Calculation of thermodynamic quantities}

The equilibrium formation energy and volume data for all the phases considered in our analysis using the LAE were retrieved from the OQMD [28,29]. The data set consists of DFT-calculated properties of over 450000 compounds, which include (a) unique, ordered experimentally reported compounds from the ICSD and (b) hypothetical compounds generated by the decoration of common structural prototypes with all the elements in the periodic table. Details of the settings used to calculate the equilibrium formation energy and volume of compounds in the OQMD can be found in Ref. [29].

All other DFT calculations reported in this work, i.e., those performed as part of global structure searches, were performed using the Vienna Ab initio Simulation Package (VASP) [102-104]. We use the projector augmented wave (PAW) formalism [105,106] and the PBE parametrization of the generalized gradient approximation to the exchangecorrelation functional [107] throughout. For all calculations, we use $\Gamma$-centered $k$-point meshes with about 8000 $k$-points per reciprocal atom and a plane-wave cutoff energy of $520 \mathrm{eV}$. All atomic and cell d.o.f. of a structure are relaxed until the force components on all the atoms are within $0.01 \mathrm{eV} / \AA$, and stresses are within a few kbar.

\section{B. Structural searches}

The minima hopping method (MHM) [108,109] implements a highly reliable algorithm to explore the low enthalpy phases of a compound at a specific pressure given solely the chemical composition [110-112]. The low-lying part of the enthalpy landscape is efficiently sampled by performing consecutive, short MD escape steps to overcome enthalpy barriers, followed by local geometry optimizations. The Bell-Evans-Polanyi principle is exploited through a feedback mechanism on the MD escape trials, and by aligning the initial MD velocities along soft-mode directions in order to accelerate the search [113,114]. The MHM has been successfully applied to identify the structure and composition of many materials, also for systems at high pressures $[12,64,65,68,115,116]$. In this work, we performed MHM simulations only at the compositions where a high-pressure phase is predicted to be stable by the linear enthalpy model.

\section{Software implementation}

All convex hull constructions in this work were performed using the Qhull library [117] with the quickhull algorithm as implemented in the SciPy PYTHON package [118]. The GCLP calculations reported in this work were performed using the $\mathrm{CBC}$ solver distributed with the PuLP PYTHON library [119]. An implementation of the framework described in Secs. I A-IC has been made available as an open-source PYTHON module [120]. An implementation of the MHM is available through the Minhocao package [108,109].

\section{ACKNOWLEDGMENTS}

M. A. and V. I. H. conceived and carried out the project, and contributed equally to this work. S. D. J. and C. W. supervised the project, and all authors contributed to writing the manuscript. M. A. (construction of linear model, crystal structure prediction) acknowledges support from the Novartis Universität Basel Excellence Scholarship for Life Sciences and the Swiss National Science Foundation (Projects No. P300P2-158407 and No. P300P2-174475). V.I. H. (model implementation, high-throughput calculations) and C.W. acknowledge support from the Department of Energy, Office of Science, Basic Energy Sciences under Grant No. DE-SC0015106. S. D. J. acknowledges support from NSF Grant No. DMR-1508577 and the Carnegie/DOE Alliance Center (CDAC). The authors acknowledge support from the Data Science Initiative at Northwestern University. The computational resources from the Swiss National Supercomputing Center in Lugano (Projects No. s499, No. s621, and No. s700), the Extreme 
Science and Engineering Discovery Environment (XSEDE) (which is supported by National Science Foundation Grant No. OCI-1053575), the Bridges System at the Pittsburgh Supercomputing Center (PSC) (which is supported by NSF Grant No. ACI-1445606), the Quest High Performance Computing Facility at Northwestern University, and the National Energy Research Scientific Computing Center (DOE: DE-AC02-05CH11231) are gratefully acknowledged. We thank Professor C. Umrigar, Professor R. Hoffmann, and Professor F. DiSalvo at Cornell University for valuable expert discussions.

[1] J. W. Gibbs, On the Equilibrium of Heterogeneous Substances, Am. J. Sci. 16, 441 (1878).

[2] S. Rosenstein and P. P. Lamy, Some Aspects of Polymorphism, American Journal of hospital pharmacy 26, 598 (1969).

[3] J.-P. Brog, C.-L. Chanez, A. Crochet, and K. M. Fromm, Polymorphism, What It Is and How to Identify It: A Systematic Review, RSC Adv. 3, 16905 (2013).

[4] J. Gopalakrishnan, Chimie Douce Approaches to the Synthesis of Metastable Oxide Materials, Chem. Mater. 7, 1265 (1995).

[5] A. Stein, S. W. Keller, and T. E. Mallouk, Turning Down the Heat: Design and Mechanism in Solid-State Synthesis, Science 259, 1558 (1993).

[6] W. Sun, S. T. Dacek, S. P. Ong, G. Hautier, A. Jain, W. D. Richards, A. C. Gamst, K. A. Persson, and G. Ceder, The Thermodynamic Scale of Inorganic Crystalline Metastability, Sci. Adv. 2, e1600225 (2016).

[7] A. R. Oganov, Modern Methods of Crystal Structure Prediction, 1st ed. (Wiley-VCH, Verlag, 2010).

[8] C. J. Pickard and R. J. Needs, Ab Initio Random Structure Searching, J. Phys. Condens. Matter 23, 053201 (2011).

[9] W. Zhang, A. R. Oganov, A. F. Goncharov, Q. Zhu, S. E. Boulfelfel, A. O. Lyakhov, E. Stavrou, M. Somayazulu, V. B. Prakapenka, and Z. Konpkov, Unexpected Stable Stoichiometries of Sodium Chlorides, Science 342, 1502 (2013).

[10] J. P. S. Walsh, S. M. Clarke, Y. Meng, S. D. Jacobsen, and D. E. Freedman, Discovery of $\mathrm{FeBi}_{2}$, ACS Cent. Sci. 2, 867 (2016).

[11] L. Zhang, Y. Wang, J. Lv, and Y. Ma, Materials Discovery at High Pressures, Nat. Rev. Mater. 2, 17005 (2017).

[12] J. A. Flores-Livas, M. Amsler, C. Heil, A. Sanna, L. Boeri, G. Profeta, C. Wolverton, S. Goedecker, and E. K. U. Gross, Superconductivity in Metastable Phases of Phosphorus-Hydride Compounds under High Pressure, Phys. Rev. B 93, 020508 (2016).

[13] T. Bi, D. P. Miller, A. Shamp, and E. Zurek, Superconducting Phases of Phosphorus Hydride under Pressure: Stabilization by Mobile Molecular Hydrogen, Angew. Chem. 129, 10326 (2017).

[14] A. Shamp, T. Terpstra, T. Bi, Z. Falls, P. Avery, and E. Zurek, Decomposition Products of Phosphine under Pressure: $\mathrm{PH}_{2}$ Stable and Superconducting?, J. Am. Chem. Soc. 138, 1884 (2016).
[15] H. Liu, Y. Li, G. Gao, J. S. Tse, and I. I. Naumov, Crystal Structure and Superconductivity of $\mathrm{PH}_{3}$ at High Pressures, J. Phys. Chem. C 120, 3458 (2016).

[16] J. A. Flores-Livas, A. Sanna, and E. K. U. Gross, High Temperature Superconductivity in Sulfur and Selenium Hydrides at High Pressure, Eur. Phys. J. B 89, 63 (2016).

[17] Y. Li, J. Hao, H. Liu, Y. Li, and Y. Ma, The Metallization and Superconductivity of Dense Hydrogen Sulfide, J. Chem. Phys. 140, 174712 (2014).

[18] M. Amsler, S. Botti, M. A. L. Marques, T. J. Lenosky, and S. Goedecker, Low-Density Silicon Allotropes for Photovoltaic Applications, Phys. Rev. B 92, 014101 (2015).

[19] R. Rasoulkhani, H. Tahmasbi, S. A. Ghasemi, S. Faraji, S. Rostami, and M. Amsler, Energy Landscape of $\mathrm{ZnO}$ Clusters and Low-Density Polymorphs, Phys. Rev. B 96, 064108 (2017).

[20] Y. Wang, M. Miao, J. Lv, L. Zhu, K. Yin, H. Liu, and Y. Ma, An Effective Structure Prediction Method for Layered Materials Based on 2D Particle Swarm Optimization Algorithm, J. Chem. Phys. 137, 224108 (2012).

[21] X.-F. Zhou, X. Dong, A. R. Oganov, Q. Zhu, Y. Tian, and H.-T. Wang, Semimetallic Two-Dimensional Boron Allotrope with Massless Dirac Fermions, Phys. Rev. Lett. 112, 085502 (2014).

[22] H. A. Eivari, S. A. Ghasemi, H. Tahmasbi, S. Rostami, S. Faraji, R. Rasoulkhani, S. Goedecker, and M. Amsler, Two-Dimensional Hexagonal Sheet of $\mathrm{TiO}_{2}$, Chem. Mater. 29, 8594 (2017).

[23] A. L.-S. Chua, N. A. Benedek, L. Chen, M. W. Finnis, and A.P. Sutton, A Genetic Algorithm for Predicting the Structures of Interfaces in Multicomponent Systems, Nat. Mater. 9, 418 (2010).

[24] G. Schusteritsch and C. J. Pickard, Predicting Interface Structures: From $\mathrm{SrTiO}_{3}$ to Graphene, Phys. Rev. B 90 , 035424 (2014).

[25] S. Lu, Y. Wang, H. Liu, M.-s. Miao, and Y. Ma, Selfassembled Ultrathin Nanotubes on Diamond (100) Surface, Nat. Commun. 5, 3666 (2014).

[26] X. Zhao, Q. Shu, M. C. Nguyen, Y. Wang, M. Ji, H. Xiang, K.-M. Ho, X. Gong, and C.-Z. Wang, Interface Structure Prediction from First-Principles, J. Phys. Chem. C 118, 9524 (2014).

[27] G. Fisicaro, M. Sicher, M. Amsler, S. Saha, L. Genovese, and S. Goedecker, Surface Reconstruction of Fluorites in Vacuum and Aqueous Environment, Phys. Rev. Mater. 1, 033609 (2017).

[28] J. E. Saal, S. Kirklin, M. Aykol, B. Meredig, and C. Wolverton, Materials Design and Discovery with HighThroughput Density Functional Theory: The Open Quantum Materials Database (OQMD), JOM 65, 1501 (2013).

[29] S. Kirklin, J. E. Saal, B. Meredig, A. Thompson, J. W. Doak, M. Aykol, S. Rühl, and C. Wolverton, The Open Quantum Materials Database (OQMD): Assessing the Accuracy of DFT Formation Energies, npj Comput. Mater. 1, 15010 (2015).

[30] A. Jain, S. P. Ong, G. Hautier, W. Chen, W. D. Richards, S. Dacek, S. Cholia, D. Gunter, D. Skinner, G. Ceder, and K. Persson, The Materials Project: A Materials Genome Approach to Accelerating Materials Innovation, APL Mater. 1, 011002 (2013). 
[31] S. Curtarolo, W. Setyawan, S. Wang, J. Xue, K. Yang, R. H. Taylor, L. J. Nelson, G. L. W. Hart, S. Sanvito, M. Buongiorno-Nardelli, N. Mingo, and O. Levy, AFLOWLIB.ORG: A Distributed Materials Properties Repository from High-Throughput Ab Initio Calculations, Comput. Mater. Sci. 58, 227 (2012).

[32] S. Curtarolo, G. L. W. Hart, M. B. Nardelli, N. Mingo, S. Sanvito, and O. Levy, The High-Throughput Highway to Computational Materials Design, Nat. Mater. 12, 191 (2013).

[33] C. Toher, C. Oses, D. Hicks, E. Gossett, F. Rose, P. Nath, D. Usanmaz, D. C. Ford, E. Perim, C. E. Calderon, J. J. Plata, Y. Lederer, M. Jahntek, W. Setyawan, S. Wang, J. Xue, K. Rasch, R. V. Chepulskii, R. H. Taylor, G. Gomez et al., The AFLOW Fleet for Materials Discovery, arXiv: 1712.00422 .

[34] Inorganic Crystal Structure Database, https://icsd.fizkarlsruhe.de.

[35] G. Shen and H. K. Mao, High-Pressure Studies with X-Rays Using Diamond Anvil Cells, Rep. Prog. Phys. 80, 016101 (2017).

[36] J. Tsuchiya, First Principles Prediction of a New HighPressure Phase of Dense Hydrous Magnesium Silicates in the Lower Mantle, Geophys. Res. Lett. 40, 4570 (2013).

[37] This procedure is analogous to calculating the range of thermodynamic stability of a compound with respect to the chemical potential of a given species. Since the chemical potential of species $i$, at $p, T=0$, is given by $\mu_{i}=$ $\left[(\partial E) /\left(\partial N_{i}\right)\right]_{N_{j \neq i}}$, the window of stability $\left[\mu_{i}^{-}, \mu_{i}^{+}\right]$of the phase can be calculated using $\mu_{i}^{ \pm}=\left\{\left[E_{0}-E\left(N_{i} \mp\right.\right.\right.$ $\left.\left.\left.d N_{i}\right)\right] /\left(d N_{i}\right)\right\}$, i.e., as a perturbation of energy with respect to the composition of the species $i$.

[38] A. R. Akbarzadeh, V. Ozolin̄š, and C. Wolverton, FirstPrinciples Determination of Multicomponent Hydride Phase Diagrams: Application to the Li-Mg-N-H System, Adv. Mater. 19, 3233 (2007).

[39] T. H. Mason, X. Liu, J. Hong, J. Graetz, and E. H. Majzoub, First-Principles Study of Novel Conversion Reactions for High-Capacity Li-Ion Battery Anodes in the Li-Mg-B-N-H System, J. Phys. Chem. C 115, 16681 (2011).

[40] D. S. Aidhy, Y. Zhang, and C. Wolverton, Prediction of a $\mathrm{Ca}\left(\mathrm{BH}_{4}\right)\left(\mathrm{NH}_{2}\right)$ Quaternary Hydrogen Storage Compound from First-Principles Calculations, Phys. Rev. B 84, 134103 (2011).

[41] S. Kirklin, B. Meredig, and C. Wolverton, High-Throughput Computational Screening of New Li-Ion Battery Anode Materials, Adv. Energy Mater. 3, 252 (2013).

[42] A. A. Emery, J. E. Saal, S. Kirklin, V. I. Hegde, and C. Wolverton, High-Throughput Computational Screening of Perovskites for Thermochemical Water Splitting Applications, Chem. Mater. 28, 5621 (2016).

[43] J. Ma, V. I. Hegde, K. Munira, Y. Xie, S. Keshavarz, D. T. Mildebrath, C. Wolverton, A. W. Ghosh, and W. H. Butler, Computational Investigation of Half-Heusler Compounds for Spintronics Applications, Phys. Rev. B 95, 024411 (2017).

[44] B. N. Dutta, Lattice Constants and Thermal Expansion of Silicon up to $900^{\circ}$ by X-ray Method, Phys. Status Solidi (b) 2, 984 (1962).
[45] J. C. Jamieson, Crystal Structures at High Pressures of Metallic Modifications of Silicon and Germanium, Science 139, 762 (1963).

[46] M. I. McMahon and R. J. Nelmes, New High-Pressure Phase of Si, Phys. Rev. B 47, 8337 (1993).

[47] J.Z. Hu and I. L. Spain, Phases of Silicon at High Pressure, Solid State Commun. 51, 263 (1984).

[48] M. Hanfland, U. Schwarz, K. Syassen, and K. Takemura, Crystal Structure of the High-Pressure Phase Silicon VI, Phys. Rev. Lett. 82, 1197 (1999).

[49] H. Olijnyk, S. K. Sikka, and W. B. Holzapfel, Structural Phase Transitions in Si and Ge under Pressures up to 50 GPa, Phys. Lett. A 103, 137 (1984).

[50] S. J. Duclos, Y. K. Vohra, and A. L. Ruoff, HCP to FCC Transition in Silicon at $78 \mathrm{GPa}$ and Studies to $100 \mathrm{GPa}$, Phys. Rev. Lett. 58, 775 (1987).

[51] C. L. Fu and K. M. Ho, First-Principles Calculation of the Equilibrium Ground-State Properties of Transition Metals: Applications to Nb and Mo, Phys. Rev. B 28, 5480 (1983).

[52] R. Evarestov, Quantum Chemistry of Solids: The LCAO First Principles Treatment of Crystals, Springer Series in Solid-State Sciences (Springer-Verlag, Berlin, Heidelberg, 2007).

[53] R. M. Brugger, R. B. Bennion, and T. G. Worlton, The Crystal Structure of Bismuth-II at 26 kbar, Phys. Lett. A 24, 714 (1967).

[54] L. G. Akselrud, M. Hanfland, and U. Schwarz, Refinement of the Crystal Structure of Bi-II, at 2.54 GPa, Z. Kristallogr. New Cryst. Struct. 218, 415 (2014).

[55] J. H. Chen, H. Iwasaki, T. Kikegawa, K. Yaoita, and K. Tsuji, Crystal Structure of the High Pressure Phase of Bismuth Bi-III, in AIP Conference Proceedings (AIP Publishing, 1994), Vol. 309, pp. 421-424, https://doi.org/10.1063/1 .46132 .

[56] M. I. McMahon, O. Degtyareva, and R. J. Nelmes, Ba-IV-Type Incommensurate Crystal Structure in Group-V Metals, Phys. Rev. Lett. 85, 4896 (2000).

[57] U. Häussermann, K. Söderberg, and R. Norrestam, Comparative Study of the High-Pressure Behavior of As, Sb, and Bi, J. Am. Chem. Soc. 124, 15359 (2002).

[58] J. H. Chen, H. Iwasaki, and T. Kikegawa, Structural Study of the High-Pressure High-Temperature Phase of Bismuth Using High Energy Synchrotron Radiation, J. Phys. Chem. Solids 58, 247 (1997).

[59] K. Aoki, S. Fujiwara, and M. Kusakabe, Stability of the bcc Structure of Bismuth at High Pressure, J. Phys. Soc. Jpn. 51, 3826 (1982).

[60] J. Sun, R. C. Remsing, Y. Zhang, Z. Sun, A. Ruzsinszky, H. Peng, Z. Yang, A. Paul, U. Waghmare, X. Wu, M. L. Klein, and J. P. Perdew, Accurate First-Principles Structures and Energies of Diversely Bonded Systems from an Efficient Density Functional, Nat. Chem. 8, 831 (2016).

[61] R. G. Hennig, A. Wadehra, K. P. Driver, W. D. Parker, C. J. Umrigar, and J. W. Wilkins, Phase Transformation in Si from Semiconducting Diamond to Metallic $\beta$-Sn Phase in QMC and DFT under Hydrostatic and Anisotropic Stress, Phys. Rev. B 82, 014101 (2010).

[62] On our HPC infrastructure, the explicit calculation of the enthalpies at different pressures for all relevant $\mathrm{Si}$ phases requires approximately 1120 core hours. This is by far the 
most expensive but also the most accurate approach since all d.o.f. (atomic coordinates and cell parameters) are fully relaxed at relevant pressures. The LAE, on the other hand, only requires the relaxed cell parameters and energies at $0 \mathrm{GPa}$, which are computed within approximately 70 core hours. For fitting the EOS, these relaxed cells are scaled to discrete volumes and the corresponding energies calculated with single-point DFT calculations, a task completed within approximately $70+140=210$ core hours (i.e., including the initial relaxation at $0 \mathrm{GPa}$ ).

[63] S. M. Clarke, J. P. S. Walsh, M. Amsler, C. D. Malliakas, T. Yu, S. Goedecker, Y. Wang, C. Wolverton, and D. E. Freedman, Discovery of a Superconducting Cu-Bi Intermetallic Compound by High-Pressure Synthesis, Angew. Chem., Int. Ed. Engl. 55, 13446 (2016).

[64] S. M. Clarke, M. Amsler, J. P. S. Walsh, T. Yu, Y. Wang, Y. Meng, S. D. Jacobsen, C. Wolverton, and D. E. Freedman, Creating Binary $\mathrm{Cu}-\mathrm{Bi}$ Compounds via High-Pressure Synthesis: A Combined Experimental and Theoretical Study, Chem. Mater. 29, 5276 (2017).

[65] M. Amsler, S. S. Naghavi, and C. Wolverton, Prediction of Superconducting Iron-Bismuth Intermetallic Compounds at High Pressure, Chem. Sci. 8, 2226 (2017).

[66] K. M. Powderly, S. M. Clarke, M. Amsler, C. Wolverton, C. D. Malliakas, Y. Meng, S. D. Jacobsen, and D. E. Freedman, High-Pressure Discovery of $\beta$-NiBi, Chem. Commun. 53, 11241 (2017).

[67] M. Amsler, Z. Yao, and C. Wolverton, Cubine, a Quasi Two-Dimensional Copper-Bismuth Nano Sheet, Chem. Mater. 29, 9819 (2017).

[68] M. Amsler and C. Wolverton, Dense Superconducting Phases of Copper-Bismuth at High Pressure, Phys. Rev. Mater. 1, 031801 (2017).

[69] G. Haegg and G. Funke, Roentgenalanlyse des Systems Nickel-Wismut, Z. Phys. Chem., Abt. B 6, 272 (1929).

[70] V. P. Glagoleva and G. S. Zhdanov, Struktura Sverkhprovodnikov. 7. Rentgenograficheskoe Opredelenie Struktury $\mathrm{Bi}_{3} \mathrm{Ni}, \mathrm{Zh}$. Eksp. Teor. Fiz. 26, 337 (1954).

[71] M. Ruck and T. Söhnel, Transmissionsoptimierte Einkristallstrukturbestimmung und Elektronische Struktur von $\mathrm{Bi}_{3} \mathrm{Ni}$, Z. Naturforsch. 61b, 785 (2006).

[72] N. E. Alekseevskii, N. B. Brandt, and T. I. Kostina, Superconductivity of Binary Alloys of Bismuth, Izv. Akad. Nauk SSSR 16, 233 (1952).

[73] N. E. Alekseevskii, Sverkhprovodimost Soedinenii Vismuta, Zh. Eksp. Teor. Fiz. 18, 101 (1948).

[74] T. Herrmannsdörfer, R. Skrotzki, J. Wosnitza, D. Köhler, R. Boldt, and M. Ruck, Structure-Induced Coexistence of Ferromagnetic and Superconducting States of SinglePhase $\mathrm{Bi}_{3} \mathrm{Ni}$ Seen via Magnetization and Resistance Measurements, Phys. Rev. B 83, 140501 (2011).

[75] F. Bachhuber, J. Rothballer, T. Söhnel, and R. Weihrich, Phase Stabilities at a Glance: Stability Diagrams of Nickel Dipnictides, J. Chem. Phys. 139, 214705 (2013).

[76] See Supplemental Material at http://link.aps.org/ supplemental/10.1103/PhysRevX.8.041021 for detailed description of the curated data sets, the predicted pressure ranges of stability of the elemental high-pressure phases, an analysis of the high-pressure stability of binary phases, further details on the $\mathrm{Ni}-\mathrm{Bi}$ phase diagram, and the predicted structures in selected immiscible, binary intermetallics.

[77] C. S. Barrett, L. Meyer, and J. Wasserman, Antiferromagnetic and Crystal Structures of Alpha-Oxygen, J. Chem. Phys. 47, 592 (1967).

[78] M. Nicol and K. Syassen, High-Pressure Optical Spectra of Condensed Oxygen, Phys. Rev. B 28, 1201 (1983).

[79] L. F. Lundegaard, G. Weck, M. I. McMahon, S. Desgreniers, and P. Loubeyre, Observation of an $\mathrm{O}_{8}$ Molecular Lattice in the $\epsilon$ Phase of Solid Oxygen, Nature (London) 443, 201 (2006).

[80] H. Fujihisa, Y. Akahama, H. Kawamura, Y. Ohishi, O. Shimomura, H. Yamawaki, M. Sakashita, Y. Gotoh, S. Takeya, and K. Honda, $\mathrm{O}_{8}$ Cluster Structure of the Epsilon Phase of Solid Oxygen, Phys. Rev. Lett. 97, 085503 (2006).

[81] Y. Ma, A. R. Oganov, and C. W. Glass, Structure of the Metallic $\zeta$-Phase of Oxygen and Isosymmetric Nature of the $\epsilon-\zeta$ Phase Transition: Ab Initio Simulations, Phys. Rev. B 76, 064101 (2007).

[82] G. Weck, S. Desgreniers, P. Loubeyre, and M. Mezouar, Single-Crystal Structural Characterization of the Metallic Phase of Oxygen, Phys. Rev. Lett. 102, 255503 (2009).

[83] S. K. Sikka, Y. K. Vohra, and R. Chidambaram, Omega Phase in Materials, Prog. Mater. Sci. 27, 245 (1982).

[84] H. Xia, S. J. Duclos, A. L. Ruoff, and Y. K. Vohra, New High-Pressure Phase Transition in Zirconium Metal, Phys. Rev. Lett. 64, 204 (1990).

[85] S. Desgreniers and K. Lagarec, High-Density $\mathrm{ZrO}_{2}$ and $\mathrm{HfO}_{2}$ : Crystalline Structures and Equations of State, Phys. Rev. B 59, 8467 (1999).

[86] O. Ohtaka, T. Nagai, T. Yamanaka, T. Yagi, and O. Shimomura, In Situ Observation of $\mathrm{ZrO}_{2}$ Phases at High Pressure and Temperature, in Properties of Earth and Planetary Materials at High Pressure and Temperature (American Geophysical Union, Washington, DC, 2013), pp. 429-433.

[87] N. Schönberg, The Structure of the Metallic Quaternary Phase ZrTaNO., Acta Chem. Scand. 8, 627 (1954).

[88] A. S. Cooper, Precise Lattice Constants of Germanium, Aluminum, Gallium Arsenide, Uranium, Sulphur, Quartz and Sapphire, Acta Crystallogr. 15, 578 (1962).

[89] S. B. Qadri, E. F. Skelton, and A. W. Webb, High Pressure Studies of Ge Using Synchrotron Radiation, J. Appl. Phys. 54, 3609 (1983).

[90] Y. K. Vohra, K. E. Brister, S. Desgreniers, A. L. Ruoff, K. J. Chang, and M. L. Cohen, Phase-Transition Studies of Germanium to 1.25 Mbar, Phys. Rev. Lett. 56, 1944 (1986).

[91] K. Takemura, U. Schwarz, K. Syassen, N. E. Christensen, M. Hanfland, D. L. Novikov, and I. Loa, High-Pressure Structures of Ge above 100 GPa, Phys. Status Solidi (b) 223, 385 (2001).

[92] K. Takemura, U. Schwarz, K. Syassen, M. Hanfland, N. E. Christensen, D. L. Novikov, and I. Loa, High-Pressure Cmca and hcp Phases of Germanium, Phys. Rev. B 62, R10603 (2000).

[93] E. Yu Tonkov and E. G. Ponyatovsky, Phase Transformations of Elements under High Pressure, 1st ed. (CRC Press, Boca Raton, FL, 2004). 
[94] A. Mujica, S. Radescu, A. Muoz, and R. J. Needs, HighPressure Phases of Germanium, J. Phys. Condens. Matter 13, 35 (2001).

[95] J. Haines, O. Cambon, E. Philippot, L. Chapon, and S. Hull, A Neutron Diffraction Study of the Thermal Stability of the $\alpha$-Quartz-Type Structure in Germanium Dioxide, J. Solid State Chem. 166, 434 (2002).

[96] N. S. Brar and H. H. Schloessin, The Kinetics of the $\mathrm{GeO}_{2}$ $(\alpha$-Quartz $) \rightarrow$ (Rutile) Transformation under High Pressure, High Temp. High Press. 13, 313 (1981).

[97] J. Haines, J. M. Lger, C. Chateau, and A. S. Pereira, Structural Evolution of Rutile-Type and CaCl-Type Germanium Dioxide at High Pressure, Phys. Chem. Miner. 27, 575 (2000).

[98] K. Shiraki, T. Tsuchiya, and S. Ono, Structural Refinements of High-Pressure Phases in Germanium Dioxide, Acta Crystallogr. Sect. D 59, 701 (2003).

[99] H. Fujihisa, Y. Akahama, H. Kawamura, Y. Ohishi, Y. Gotoh, H. Yamawaki, M. Sakashita, S. Takeya, and K. Honda, Incommensurate Structure of Phosphorus Phase IV, Phys. Rev. Lett. 98, 175501 (2007).

[100] S. Kirklin, J. E. Saal, V. I. Hegde, and C. Wolverton, HighThroughput Computational Search for Strengthening Precipitates in Alloys, Acta Mater. 102, 125 (2016).

[101] T. B. Massalski, H. Okamoto, P. R. Subramanian, L. Kacprzak, and W. W. Scott, Binary Alloy Phase Diagrams (American Society for Metals, Metals Park, OH, 1986), Vol. 1.

[102] G. Kresse and J. Hafner, Ab Initio Molecular Dynamics for Liquid Metals, Phys. Rev. B 47, 558 (1993).

[103] G. Kresse and J. Furthmüller, Efficiency of Ab-Initio Total Energy Calculations for Metals and Semiconductors Using a Plane-Wave Basis Set, Comput. Mater. Sci. 6, 15 (1996).

[104] G. Kresse and J. Furthmüller, Efficient Iterative Schemes for Ab Initio Total-Energy Calculations Using a PlaneWave Basis Set, Phys. Rev. B 54, 11169 (1996).

[105] P. E. Blöchl, Projector Augmented-Wave Method, Phys. Rev. B 50, 17953 (1994).

[106] G. Kresse and D. Joubert, From Ultrasoft Pseudopotentials to the Projector Augmented-Wave Method, Phys. Rev. B 59, 1758 (1999).

[107] J. P. Perdew, K. Burke, and M. Ernzerhof, Generalized Gradient Approximation Made Simple, Phys. Rev. Lett. 77, 3865 (1996).
[108] S. Goedecker, Minima Hopping: An Efficient Search Method for the Global Minimum of the Potential Energy Surface of Complex Molecular Systems, J. Chem. Phys. 120, 9911 (2004).

[109] M. Amsler and S. Goedecker, Crystal Structure Prediction Using the Minima Hopping Method, J. Chem. Phys. 133, 224104 (2010).

[110] M. Amsler, J. A. Flores-Livas, T. D. Huan, S. Botti, M. A. L. Marques, and S. Goedecker, Novel Structural Motifs in Low Energy Phases of $\mathrm{LiAlH}_{4}$, Phys. Rev. Lett. 108, 205505 (2012).

[111] T. D. Huan, M. Amsler, V. N. Tuoc, A. Willand, and S. Goedecker, Low-Energy Structures of Zinc Borohydride $\mathrm{Zn}\left(\mathrm{BH}_{4}\right)_{2}$, Phys. Rev. B 86, 224110 (2012).

[112] T. D. Huan, M. Amsler, R. Sabatini, V. N. Tuoc, N. B. Le, L. M. Woods, N. Marzari, and S. Goedecker, Thermodynamic Stability of Alkali-Metal-Zinc Double-Cation Borohydrides at Low Temperatures, Phys. Rev. B 88, 024108 (2013).

[113] S. Roy, S. Goedecker, and V. Hellmann, Bell-EvansPolanyi Principle for Molecular Dynamics Trajectories and Its Implications for Global Optimization, Phys. Rev. E 77, 056707 (2008).

[114] M. Sicher, S. Mohr, and S. Goedecker, Efficient Moves for Global Geometry Optimization Methods and Their Application to Binary Systems, J. Chem. Phys. 134, 044106 (2011).

[115] M. Amsler, J. A. Flores-Livas, L. Lehtovaara, F. Balima, S. A. Ghasemi, D. Machon, S. Pailhès, A. Willand, D. Caliste, S. Botti, A. San Miguel, S. Goedecker, and M. A. L. Marques, Crystal Structure of Cold Compressed Graphite, Phys. Rev. Lett. 108, 065501 (2012).

[116] J. A. Flores-Livas, M. Amsler, T. J. Lenosky, L. Lehtovaara, S. Botti, M. A. L. Marques, and S. Goedecker, HighPressure Structures of Disilane and Their Superconducting Properties, Phys. Rev. Lett. 108, 117004 (2012).

[117] C. B. Barber, D. P. Dobkin, and H. Huhdanpaa, The Quickhull Algorithm for Convex Hulls, ACM Trans. Math. Softw. 22, 469 (1996).

[118] E. Jones, T. Oliphant, P. Peterson et al., SciPy: Open Source Scientific Tools for Python, 2001, https://www .scipy.org/citing.html.

[119] S. Mitchell, M. O'Sullivan, and I. Dunning, PuLP: A Linear Programming Toolkit for Python (2011).

[120] V.I. Hegde and M. Amsler, wolverton-research-group/ nve, 2017, https://doi.org/10.5281/zenodo.1001809. 